\title{
Improved Analysis of GW150914 Using a Fully Spin-Precessing Waveform Model
}

\author{
B. P. Abbott et al. \\ (LIGO Scientific Collaboration and Virgo Collaboration) \\ (Received 4 June 2016; revised manuscript received 30 July 2016; published 21 October 2016)
}

\begin{abstract}
This paper presents updated estimates of source parameters for GW150914, a binary black-hole coalescence event detected by the Laser Interferometer Gravitational-wave Observatory (LIGO) in 2015 [Abbott et al. Phys. Rev. Lett. 116, 061102 (2016).]. Abbott et al. [Phys. Rev. Lett. 116, 241102 (2016).] presented parameter estimation of the source using a 13-dimensional, phenomenological precessing-spin model (precessing IMRPhenom) and an 11-dimensional nonprecessing effective-onebody (EOB) model calibrated to numerical-relativity simulations, which forces spin alignment (nonprecessing EOBNR). Here, we present new results that include a 15-dimensional precessingspin waveform model (precessing EOBNR) developed within the EOB formalism. We find good agreement with the parameters estimated previously [Abbott et al. Phys. Rev. Lett. 116, 241102 (2016).], and we quote updated component masses of $35_{-3}^{+5} \mathrm{M}_{\odot}$ and $30_{-4}^{+3} \mathrm{M}_{\odot}$ (where errors correspond to $90 \%$ symmetric credible intervals). We also present slightly tighter constraints on the dimensionless spin magnitudes of the two black holes, with a primary spin estimate $<0.65$ and a secondary spin estimate $<0.75$ at $90 \%$ probability. Abbott et al. [Phys. Rev. Lett. 116, 241102 (2016).] estimated the systematic parameter-extraction errors due to waveform-model uncertainty by combining the posterior probability densities of precessing IMRPhenom and nonprecessing EOBNR. Here, we find that the two precessing-spin models are in closer agreement, suggesting that these systematic errors are smaller than previously quoted.
\end{abstract}

DOI: 10.1103/PhysRevX.6.041014

\section{INTRODUCTION}

The detection of the first gravitational-wave (GW) transient, GW150914, by the Laser Interferometer Gravitational-wave Observatory in 2015 [1] marked the beginning of a new kind of astronomy, fundamentally different from electromagnetic or particle astronomy. GW150914 was analyzed using the most accurate signal models available at the time of observation, which were developed under the assumption that general relativity is the correct theory of gravity. The analysis concluded that GW150914 was generated by the coalescence of two black holes (BHs) of rest-frame masses $36_{-4}^{+5} \mathrm{M}_{\odot}$ and $29_{-4}^{+4} \mathrm{M}_{\odot}$, at a luminosity distance of $410_{-180}^{+160} \mathrm{Mpc}$ [2]. Throughout this paper, we quote parameter estimates as the median of their posterior probability density, together with the width of the 90\% symmetric credible interval.

The GW signal emitted by a binary black hole $(\mathrm{BBH})$ depends on 15 independent parameters: the $\mathrm{BH}$ masses and the BH spin vectors (the intrinsic parameters); the inclination and the phase of the observer in the orbital plane, the sky location of the binary (parametrized by two angles, the

*Full author list given at the end of the article.

Published by the American Physical Society under the terms of the Creative Commons Attribution 3.0 License. Further distribution of this work must maintain attribution to the author(s) and the published article's title, journal citation, and DOI.
Subject Areas: Astrophysics, Gravitation

right ascension and declination), the polarization angle of the GW, the luminosity distance of the binary, and the time of arrival of the GW at the detector (all of which are known as extrinsic parameters). The task of extracting all 15 parameters from interferometric detector data relies on efficient Bayesian inference algorithms and on the availability of accurate theoretical predictions of the GW signal. State-of-the-art numerical-relativity (NR) simulations [3-8] can generate very accurate $\mathrm{BBH}$ waveforms over a large region of parameter space; however, this region does not yet include (i) binary configurations that have large dimensionless spins $(>0.5)$, extreme mass ratios $(<1 / 3)$, and many GW cycles $(\geq 40-60)$, except for a few cases [8-10]; nor does it include (ii) systems undergoing significant spin-induced precession of the orbital plane. In practice, parameter estimation requires very many waveform evaluations that span a large region of parameter space, and a purely NR approach is possible if one coarsely discretizes the intrinsic parameters, as has been done for GW150914 [11], or constructs interpolants (surrogates) across NR simulations [12]. However, a continuous sampling of the intrinsic parameter space, even outside regions where NR runs are available, is unfeasible.

The first parameter-estimation study of GW150914 [2] used two such models: an effective-one-body (EOB, Refs. [13,14]) model that restricts spins to be aligned with the orbital angular momentum [15], and a phenomenological model that includes spin-precession 
effects governed by four effective spin parameters [16]. Here, we present updated parameter estimates using a fully spin-precessing EOB model $[17,18]$, which is parametrized by the full set of $\mathrm{BBH}$ properties listed above, including all six $\mathrm{BH}$-spin degrees of freedom, and which reflects additional physical effects described in Sec. II. The inclusion of these effects motivates us to repeat the Bayesian analysis of GW150914 with precessing EOB waveforms. This model was not used in Ref. [2] because it requires costly timedomain integration for each set of BBH parameters; thus, not enough Monte Carlo samples had been collected by the time the study was finalized [19].

The main result of our analysis is that the two precessing models (phenomenological and EOB) are broadly consistent, showing largely overlapping 90\% credible intervals for all measured binary parameters, more so than the precessing phenomenological and nonprecessing EOB models compared in Ref. [2]. In that study, the parameter estimates obtained with those two models were combined with equal weights to provide the fiducial values quoted in Ref. [1], and they were differenced to characterize systematic errors due to waveform mismodeling. Because the two precessing models yield closer results, we are now able to report smaller combined credible intervals, as well as smaller estimated systematic errors. Nevertheless, the combined medians cited as fiducial estimates in Ref. [1] change only slightly. In addition, we find that some of the intrinsic parameters that affect $\mathrm{BBH}$ evolution, such as the in-plane combination of $\mathrm{BH}$ spins that governs precession, are constrained better using the precessing EOB model.

Because precessing-EOB waveforms are so computationally expensive to generate, we cannot match the number of Monte Carlo samples used in Ref. [2]. Thus, we carry out a careful statistical analysis to assess the errors of our summary statistics (posterior medians and credible intervals) due to the finite number of samples. We apply the same analysis to the precessing phenomenological and nonprecessing EOB models, and to their combinations. Although finite-sample errors are a factor of a few larger for the precessing EOB model than for the other two, they remain much smaller than the credible intervals, so none of our conclusions is affected. Last, as a further test of the accuracy and consistency of the two precessing models, we use them to estimate the known parameters of a GW150914-like NR waveform injected into LIGO data. The resulting posteriors are similar to those found for GW150914.

This article is organized as follows. In Sec. II, we discuss the modeling of spin effects in the BBH waveforms used in this paper. In Sec. III, we describe our analysis. We present our results in Sec. IV and our conclusions in Sec. V. Throughout the article, we adopt geometrized units, with $G=c=1$.

\section{MODELING ORBITAL PRECESSION IN BBH WAVEFORM MODELS}

Astrophysical stellar-mass BHs are known to possess significant intrinsic spins, which can engender large effects in the late phase of BBH coalescences: they affect the evolution of orbital frequency, and (if the $\mathrm{BH}$ spins are not aligned with the orbital angular momentum) they induce the precession of the orbital plane, modulating the fundamental chirping structure of emitted GWs in a manner dependent on the relative angular geometry of binary and observatory [20]. While measuring BH spins is interesting in its own right, the degree of their alignment and the resulting degree of precession hold precious clues to the astrophysical origin of stellar-mass BBHs [21]: Aligned spins suggest that the two BHs were born from an undisturbed binary star in which both components successively collapsed to BHs; nonaligned spins point to an origin from capture events and three-body interactions in dense stellar environments.

Clearly, the accurate modeling of BH-spin effects is crucial to BBH parameter-estimation studies. Now, even state-of-the-art semianalytical waveform models still rely on a set of approximations that necessarily limit their accuracy. These include finite post-Newtonian (PN) order, calibration to a limited number of NR simulations, rotation to precessing frames, and more. Thus, being able to compare parameter estimates performed with different waveform models, derived under different assumptions and approximations (e.g., in time- vs frequency-domain formulations), becomes desirable to assess the systematic biases due to waveform mismodeling. While observing consistent results does not guarantee the absence of systematic errors (after all, multiple models could be wrong in the same way), the fact that we do not observe inconsistencies does increase our confidence in the models.

Such a comparison was performed in the original parameter-estimation study of GW150914 [2], showing consistency between the precessing phenomenological model and the aligned-spin EOBNR model. This result matched the finding that the $\mathrm{BH}$ spins were approximately aligned in GW150914, or that precession effects were too weak to be detected, because of the small number of GW cycles and of the (putative) face-on/face-off presentation of the binary. Nevertheless, it may be argued that the conclusion of consistency remained suspect because only one model in the analysis carried information about the effects of precession; conversely, the estimates of mismodeling systematic errors performed in Ref. [2] were likely increased by the fact that the nonprecessing model would be biased by what little precession may be present in the signal.

The analysis presented in this article, which relies on two precessing-spin waveform families, removes both limitations and sets up a more robust framework to assess systematic biases in future detections where spin effects 
play a larger role. In the rest of this section, we discuss the features and formulation of the fully precessing EOBNR model. The reader not interested in these technical details (and in the Bayesian-inference setup of Sec. III) may proceed directly to Sec. IV.

The precessing EOBNR model (henceforth, "precessing EOBNR") used here can generate inspiral-merger-ringdown (IMR) waveforms for coalescing, quasicircular $\mathrm{BH}$ binaries with mass ratio $0.01 \leq q \equiv m_{2} / m_{1} \leq 1$, dimensionless $\mathrm{BH}$ spin magnitudes $0 \leq \chi_{1,2} \equiv\left|\boldsymbol{S}_{1,2}\right| / m_{1,2}^{2} \leq$ 0.99 , and arbitrary $\mathrm{BH}$ spin orientations [22]. We denote with $m_{1,2}$ the masses of the component objects in the binary and with $\boldsymbol{S}_{1,2}$ their spin vectors. Note that the model was calibrated only to 38 nonspinning NR simulations that span a smaller portion of the parameter space than defined above, but it was not calibrated to any precessing NR waveform (see below for more details).

The fundamental idea of EOB models consists in mapping the conservative dynamics of a binary to that of a spinning particle that moves in a deformed Kerr spacetime [13,14, 23-28], where the magnitude of the deformation is proportional to the mass ratio of the binary. This mapping can be seen as a resummation of PN formulas [29] with the aim of extending their validity to the strong-field regime. As for dissipative effects, EOB models equate the loss of energy to the GW luminosity, which is expressed as a sum of squared amplitudes of the multipolar waveform modes. In the nonprecessing limit, the inspiral-plunge waveform modes are themselves resummations of PN expressions [30-32] and are functionals of the orbital dynamics. The ringdown signal is described by a linear superposition of the quasinormal modes [33-35] of the remnant $\mathrm{BH}$.

EOB models can be tuned to NR by introducing adjustable parameters at high, unknown PN orders. For the precessing EOB model used in this work, the relevant calibration to NR was carried out in Ref. [15] against 38 NR simulations of nonprecessing-spin systems from Ref. [36], with mass ratios up to $1 / 8$ and spin magnitudes up to almost extremal for equal-mass BBHs and up to 0.5 for unequal-mass BBHs.

Furthermore, information from inspiral, merger, and ringdown waveforms in the test-particle limit were also included in the EOBNR model $[37,38]$. Prescriptions for the onset and spectrum of ringdown for precessing BBHs were first given in Ref. [17] and significantly improved in Ref. [18].

In the model, the $\mathrm{BH}$ spin vectors precess according to

$$
\frac{\mathrm{d} \boldsymbol{S}_{1,2}}{\mathrm{~d} t}=\frac{\partial H_{\mathrm{EOB}}}{\partial \boldsymbol{S}_{1,2}} \times \boldsymbol{S}_{1,2}
$$

when the $\mathrm{BH}$ spins are oriented generically, the orbital plane precesses with respect to an inertial observer. The orientation of the orbital plane is tracked by the Newtonian orbital angular momentum $\boldsymbol{L}_{\mathrm{N}} \equiv \mu \boldsymbol{r} \times \dot{\boldsymbol{r}}$, where $\mu \equiv m_{1} m_{2} /\left(m_{1}+m_{2}\right)$ and $\boldsymbol{r}$ is the relative $\mathrm{BH}$ separation. One defines a (noninertial) precessing frame whose $z$ axis is aligned with $\boldsymbol{L}_{\mathrm{N}}(t)$, and whose $x$ and $y$ axes obey the minimum-rotation prescription of Refs. [39,40]. In this frame, the waveform amplitude and phase modulations induced by precession are minimized, as pointed out in several studies [39-43].

Thus, the construction of a precessing EOB waveform consists of the following steps: (i) Compute orbital dynamics numerically, by solving Hamilton's equation for the EOB Hamiltonian, subject to energy loss, up until the lightring (or photon-orbit) crossing; (ii) generate inspiral-plunge waveforms in the precessing frame as if the system were not precessing [15]; (iii) rotate the waveforms to the inertial frame aligned with the direction of the remnant spin; (iv) generate the ringdown signal, and connect it smoothly to the inspiral-plunge signal; (v) rotate the waveforms to the inertial frame of the observer.

A phenomenological precessing-spin IMR model (henceforth, "precessing IMRPhenom") was proposed in Refs. $[16,44,45]$. These waveforms are generated in the frequency domain by rotating nonprecessing phenomenological waveforms [46] from a precessing frame to the inertial frame of the observer, according to PN formulas that describe precession in terms of Euler angles. The underlying nonprecessing waveforms depend on the $\mathrm{BH}$ masses and on the two projections of the spins on the Newtonian angular momentum, with the spin of the $\mathrm{BH}$ formed through merger adjusted to also take into account the effect of the in-plane spin components. The influence of the in-plane spin components on the precession is modeled with a single-spin parameter (a function of the two $\mathrm{BH}$ spins) and also depends on the initial phase of the binary in the orbital plane. Thus, this model only has four independent parameters to describe the 6 spin degrees of freedom, which is justified by the analysis of dominant spin effects performed in Ref. [44].

While both precessing EOBNR and IMRPhenom models describe spin effects, there are important differences in how they account for precession, which is the main focus of this paper.

(1) In precessing IMRPhenom, the precessing-frame inspiral-plunge waveforms are strictly nonprecessing waveforms, while for precessing EOBNR, some precessional effects are included (such as spin-spin frequency and amplitude modulations) since the orbital dynamics that enters the nonprecessing expressions for the GW modes is fully precessing.

(2) The precessing EOBNR merger-ringdown signal is generated in the inertial frame oriented along the total angular momentum of the remnant - the very frame where quasinormal mode frequencies are computed in $\mathrm{BH}$ perturbation theory. By contrast, precessing IMRPhenom generates the mergerringdown signal directly in the precessing frame. 
(3) The IMRPhenom precessing-frame waveforms contain only the dominant $(2, \pm 2)$ modes [47], while precessing EOBNR also includes $(2, \pm 1)$ modes in the precessing frame, although these are not calibrated to NR.

(4) In IMRPhenom, the frequency-domain rotation of the GW modes from the precessing frame to the inertial frame is based on approximate formulas (i.e., on the stationary-phase approximation), while precessing EOBNR computes the rotations fully in the time domain, where the formulas are straightforward.

(5) In precessing IMRPhenom, the frequency-domain formulas for the Euler angles that parametrize the precession of the orbital plane with respect to a fixed inertial frame involve several approximations: Inplane spin components are orbit averaged; the magnitude of the orbital angular momentum is approximated by its 2PN nonspinning expression; the evolution of frequency is approximated as adiabatic; and the PN formulas that regulate the behavior of the Euler angles at high frequencies are partially resummed. By contrast, precessing EOBNR defines these Euler angles on the basis of the completely general motion of $\boldsymbol{L}_{\mathrm{N}}(t)$; this motion is a direct consequence of the EOB dynamics, and as such, it is sensitive to the full precessional dynamics of the six spin components.

A priori, it is not obvious that these approximations will not impact parameter estimation for a generic $\mathrm{BBH}$. However, as far as GW150914 is concerned, Ref. [2] showed broadly consistent results between a precessing and a nonprecessing model; a fortiori, we should expect similar results between two precessing models. Indeed, the GW150914 binary is more probable to be face-off or face-on than edgeon with respect to the line of sight to the detector, and the component masses are almost equal [2]: Both conditions imply that subdominant modes play a minor role.

The nonprecessing models that underlie both approximants were tested against a large catalog of NR simulations $[15,46,48]$, finding a high degree of accuracy in the GW150914 parameter region. However, it is important to bear in mind that these waveform models can differ from NR outside the region in which they were calibrated, and they do not account for all possible physical effects that are relevant to generic BBHs, such us higher-order modes. Finally, neither of the two precessing models has been calibrated to any precessing NR simulation. Thus, we cannot exclude that current precessing models are affected by systematics. References $[17,18]$ compared the precessing EOBNR model to $70 \mathrm{NR}$ runs with mild precession (with mass ratios 1 to $1 / 5$, spin magnitudes up to 0.5 , generic spin orientations, and each about 15-20 orbital cycles long) finding sky-location and polarization-averaged overlaps typically above $97 \%$ without recalibration.
Since the generation of precessing EOBNR waveforms [at least in the current implementation in the LIGO Algorithm Library (LAL)] is a rather time-consuming process (see [19]), when carrying out parameter-estimation studies with this template family, we introduce a timesaving approximation at the level of the likelihood function. Namely, we marginalize over the arrival time and phase of the signal as if the waveforms contained only $(2, \pm 2)$ inertial-frame modes since in that case the marginalization can be performed analytically [49]. We have determined that the impact of this approximation is negligible by conducting a partial parameter-estimation study where we do not marginalize over the arrival time and phase. We can understand this physically for GW150914 because in a nearly face-on/face-off binary, the $(2, \pm 1)$ observer-frame modes are significantly subdominant compared to $(2, \pm 2)$ modes [50].

\section{BAYESIAN INFERENCE ANALYSIS}

For each waveform model under consideration, we estimate the posterior probability density $[51,52]$ for the $\mathrm{BBH}$ parameters, following the prescriptions of Ref. [2]. To wit, we use the LAL implementation of parallel-tempering Markov chain Monte Carlo and nested sampling [49] to sample the posterior density $p(\boldsymbol{\vartheta} \mid$ model, data $)$ as a function of the parameter vector $\boldsymbol{\vartheta}$ :

$$
p(\boldsymbol{\vartheta} \mid \text { model, data }) \propto \mathcal{L}(\text { data } \mid \boldsymbol{\vartheta}) \times p(\boldsymbol{\vartheta}) .
$$

To obtain the likelihood $\mathcal{L}($ data $\mid \boldsymbol{\vartheta})$, we first generate the GW polarizations $h_{+}\left(\boldsymbol{\vartheta}_{\text {intrinsic }}\right)$ and $h_{\times}\left(\boldsymbol{\vartheta}_{\text {intrinsic }}\right)$ according to the waveform model. We then combine the polarizations into the LIGO detector responses $h_{1,2}$ by way of the detector antenna patterns:

$$
\begin{aligned}
h_{k}(\boldsymbol{\vartheta})= & h_{+}\left(\boldsymbol{\vartheta}_{\text {intrinsic }}\right) F_{k}^{(+)}\left(\boldsymbol{\vartheta}_{\text {extrinsic }}\right) \\
& +h_{\times}\left(\boldsymbol{\vartheta}_{\text {intrinsic }}\right) F_{k}^{(\times)}\left(\boldsymbol{\vartheta}_{\text {extrinsic }}\right) .
\end{aligned}
$$

Finally, we compute the likelihood as the sampling distribution of the residuals [i.e., the detector data $d_{k}$ minus the GW response $h_{k}(\boldsymbol{\vartheta})$ ], under the assumption that these are distributed as Gaussian noise characterized by the power spectral density (PSD) of nearby data [49]:

$\mathcal{L}($ data $\mid \boldsymbol{\vartheta}) \propto \exp \left[-\frac{1}{2} \sum_{k=1,2}\left\langle h_{k}(\boldsymbol{\vartheta})-d_{k} \mid h_{k}(\boldsymbol{\vartheta})-d_{k}\right\rangle\right]$,

where $\langle\cdot \mid \cdot\rangle$ denotes the noise-weighted inner product [53].

The prior probability density $p(\boldsymbol{\vartheta})$ follows the choices of Ref. [2]. In particular, we assume uniform mass priors $m_{1,2} \in[10,80] \mathbf{M}_{\odot}$, with the constraint $m_{2} \leq m_{1}$, and uniform spin-amplitude priors $a_{1,2}=\left|\boldsymbol{S}_{1,2}\right| / m_{1,2}^{2} \in[0,1]$, with spin directions distributed uniformly on the two-sphere; 
and we assume that sources are distributed uniformly in Euclidian volume, with their orbital orientation distributed uniformly on the two-sphere. All the binary parameters that evolve during the inspiral (such as tilt angles between the spins and the orbital angular momentum, $\theta_{L S_{1,2}}$ ) are defined at a reference $\mathrm{GW}$ frequency $f_{\text {ref }}=20 \mathrm{~Hz}$. Following Ref. [2], we marginalize over the uncertainty in the calibration of LIGO data [54]. This broadens the posteriors but reduces calibration biases.

To assess whether the data are informative with regard to a source parameter (i.e., where it updates the prior significantly), we perform a Kolmogorov-Smirnov (KS) test. Given an empirical distribution (in our case, the Monte Carlo posterior samples) and a probability distribution (in our case, the prior), the KS test measures the maximum deviation between the two cumulative distributions and associates a $p$-value to that: For samples generated from the probability distribution against which the test is performed, one expects a $p$-value around 0.5 ; $p$ values smaller than 0.05 indicate that the samples come from a different probability distribution with a high level of significance - that is, there is only a 5\% (or less) chance that the two sets of samples come from the same distribution. The outcomes of our KS tests are only statements about how much the posteriors deviate from the respective priors, but they do not tell us anything about the astrophysical relevance of $90 \%$ credible intervals.

\section{RESULTS}

The first question that we address is whether parameter estimates derived using the two precessing models (precessing IMRPhenom and precessing EOBNR) are compatible. In particular, we compare posterior medians and $90 \%$ credible intervals (the summary statistics used in Ref. [2]) for the parameters tabulated in Table I of Ref. [2], as well as additional spin parameters. The nominal values of the medians and 5\% and 95\% quantiles for the two models are listed in the "EOBNR" and "IMRPhenom" columns of Table I and Fig. 1. However, it is unclear a priori whether any differences are due to the models themselves or to the imperfect sampling of the posteriors in Markov chain Monte Carlo runs. This is a concern especially for the precessing EOBNR results since the slower speed of EOBNR waveform generation means that shorter chains are available for parameter estimation. To gain trust in our comparisons, we characterize the Monte Carlo error of the medians and quantiles by a bootstrap analysis, as follows.

The Monte Carlo runs for the precessing IMRPhenom model produced an equal-weight posterior sampling

TABLE I. Median values of source parameters of GW150914 as estimated with the two precessing waveform models and with an equal-weight average of posteriors (in the "Overall" column). The models are described in the text. Subscripts and superscripts indicate the range of the symmetric $90 \%$ credible intervals. When useful, we quote $90 \%$ credible bounds.

\begin{tabular}{lccc}
\hline \hline & Precessing EOBNR & Precessing IMRPhenom & Overall \\
\hline Detector-frame total mass $M / \mathrm{M}_{\odot}$ & $71.6_{-4.1}^{+4.3}$ & $70.9_{-3.9}^{+4.0}$ & $71.3_{-4.1}^{+4.3}$ \\
Detector-frame chirp mass $\mathcal{M} / \mathrm{M}_{\odot}$ & $30.9_{-1.9}^{+2.0}$ & $30.6_{-1.8}^{+1.8}$ & $30.8_{-1.8}^{+1.9}$ \\
Detector-frame primary mass $m_{1} / \mathrm{M}_{\odot}$ & $38.9_{-3.7}^{+5.1}$ & $38.5_{-3.6}^{+5.6}$ & $38.7_{-3.7}^{+5.3}$ \\
Detector-frame secondary mass $m_{2} / \mathrm{M}_{\odot}$ & $32.7_{-4.8}^{+3.6}$ & $32.2_{-4.8}^{+3.6}$ & $32.5_{-4.8}^{+3.7}$ \\
Detector-frame final mass $M_{\mathrm{f}} / \mathrm{M}_{\odot}$ & $68.3_{-3.7}^{+3.8}$ & $67.6_{-3.5}^{+3.6}$ & $68.0_{-3.6}^{+3.8}$ \\
Source-frame total mass $M^{\text {source }} / \mathrm{M}_{\odot}$ & $65.6_{-3.8}^{+4.1}$ & $65.0_{-3.6}^{+4.0}$ & $65.3_{-3.7}^{+4.1}$ \\
Source-frame chirp mass $\mathcal{M}^{\text {source }} / \mathrm{M}_{\odot}$ & $28.3_{-1.7}^{+1.8}$ & $28.1_{-1.6}^{+1.7}$ & $28.2_{-1.7}^{+1.8}$ \\
Source-frame primary mass $m_{1}^{\text {source }} / \mathrm{M}_{\odot}$ & $35.6_{-3.4}^{+4.8}$ & $35.3_{-3.4}^{+5.2}$ & $35.4_{-3.4}^{+5.0}$ \\
Source-frame secondary mass $m_{2}^{\text {source }} / \mathrm{M}_{\odot}$ & $30.0_{-4.4}^{+3.3}$ & $29.6_{-4.3}^{+3.3}$ & $29.8_{-4.3}^{+3.3}$ \\
Source-frame final mass $M_{\mathrm{f}}^{\text {source }} / \mathrm{M}_{\odot}$ & $62.5_{-3.4}^{+3.7}$ & $62.0_{-3.3}^{+3.7}$ & $62.2_{-3.4}^{+3.7}$ \\
Mass ratio $q$ & $0.84_{-0.20}^{+0.14}$ & $0.84_{-0.20}^{+0.14}$ & $0.84_{-0.20}^{+0.14}$ \\
Effective inspiral spin parameter $\chi_{\text {eff }}$ & $-0.02_{-0.16}^{+0.14}$ & $-0.05_{-0.15}^{+0.13}$ & $-0.04_{-0.16}^{+0.14}$ \\
Effective precession spin parameter $\chi_{\mathrm{p}}$ & $0.28_{-0.21}^{+0.38}$ & $0.35_{-0.27}^{+0.45}$ & $0.31_{-0.23}^{+0.44}$ \\
Dimensionless primary spin magnitude $a_{1}$ & $0.22_{-0.20}^{+0.43}$ & $0.32_{-0.29}^{+0.53}$ & $0.26_{-0.24}^{+0.52}$ \\
Dimensionless secondary spin magnitude $a_{2}$ & $0.29_{-0.27}^{+0.52}$ & $0.34_{-0.31}^{+0.54}$ & $0.32_{-0.29}^{+0.54}$ \\
Final spin $a_{\mathrm{f}}$ & $0.68_{-0.05}^{+0.05}$ & $0.68_{-0.06}^{+0.06}$ & $0.68_{-0.06}^{+0.05}$ \\
Luminosity distance $D_{\mathrm{L}} / \mathrm{Mpc}$ & $440_{-180}^{+160}$ & $440_{-180}^{+150}$ & $440_{-180}^{+160}$ \\
Source redshift $z$ & $0.094_{-0.037}^{+0.032}$ & $0.093_{-0.036}^{+0.029}$ & $0.093_{-0.036}^{+0.030}$ \\
Upper bound on primary spin magnitude $a_{1}$ & 0.54 & 0.74 & 0.65 \\
Upper bound on secondary spin magnitude $a_{2}$ & 0.70 & 0.78 & 0.75 \\
Lower bound on mass ratio $q$ & 0.69 & 0.68 & 0.68 \\
\hline \hline
\end{tabular}




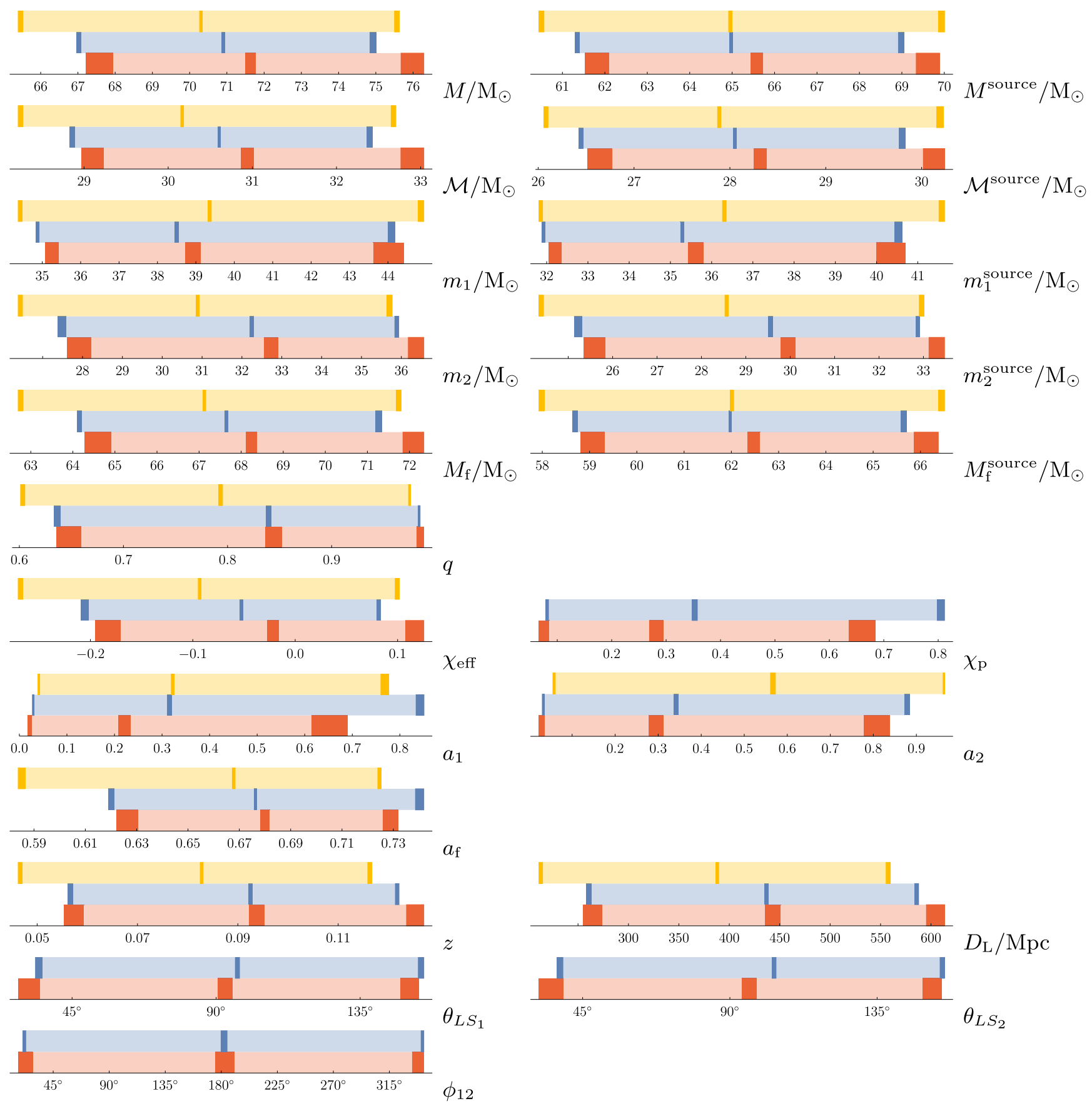

FIG. 1. Comparing nonprecessing EOBNR (light yellow, top), precessing IMRPhenom (light blue, middle), and precessing EOBNR (light red, bottom) $90 \%$ credible intervals for select GW150914 source parameters. The darker intervals represent error estimates for (from left to right) the $5 \%, 50 \%$, and $95 \%$ quantiles, estimated by Bayesian bootstrapping.

consisting of 27000 approximately independent samples, obtained by downsampling the original MCMC run by a factor equal to the largest autocorrelation length measured for the parameters of interest (those of Table I). We generate 1000 Bayesian-bootstrap weighted resamplings [55] of the equal-weight population [56], and for each, we compute the weighted medians and quantiles. We characterize the Monte Carlo error of these summary statistics as the $90 \%$ symmetric interquantile interval across the 1000 realizations. For completeness, we apply the same analysis to the 45000 samples of the nonprecessing EOBNR that were employed in Ref. [2].

The Monte Carlo runs for the precessing EOBNR model produced a sampling of 2700 approximately independent samples, obtained by selecting every 1100th sample in the original MCMC run. Again, we generate 1000 Bayesian- 
bootstrap resamplings, compute summary statistics on each, and measure their variation. However, to improve the representativeness of this analysis given the smaller number of samples in play, we use nine additional equalweight populations, obtained by selecting every $(1100+i)$ th sample in the original MCMC run, for $i=1, \ldots, 9$. For each of the 1000 Bayesian-bootstrap resamplings, we first choose randomly among the ten equal-weight populations.

Monte Carlo errors are expected to shrink as the inverse square root of the number of samples; this is indeed what we observe, with precessing EOBNR finite-sample errors about $(27000 / 2700)^{1 / 2} \approx 3$ times larger than for precessing IMRPhenom. Table I and Fig. 1 present the results of this study for several key physical parameters of the source of GW150914. With darker colors, we display the finitesample error estimates on the position of the medians and $5 \%$ and $95 \%$ quantiles. Lighter colors represent the $90 \%$ credible intervals.

Combined estimates.-To account for waveform-mismodeling errors in its fiducial parameter estimates, Ref. [2] cited quantiles for combined posteriors obtained by averaging the posteriors for its two models (in Bayesian terms, this corresponds to assuming that the observed GW signal could have come from either model with equal posterior probability). We repeat the same procedure for the two precessing models, and we show the resulting estimates in the column "Overall" of Table I. Quantiles are more uncertain for the precessing combination because of the larger finite-sampling error of precessing EOBNR. Nevertheless, 90\% credible intervals are slightly tighter than cited in Ref. [2]. In the Appendix, we provide a graphical representation of the combined estimates.

Posterior histograms: Masses and spin magnitudes.We now discuss in some detail the salient features of parameter posteriors. In Figs. 2-6, we show the onedimensional marginalized posteriors for selected pairs of parameters and $90 \%$ credible intervals (the dashed lines), as obtained for the two precessing models, as well as the twodimensional probability density plots for the precessing EOBNR model. In Fig. 2, we show the posteriors for the source-frame $\mathrm{BH}$ masses $m_{1,2}$ : These are measured fairly well, with statistical uncertainties around 10\%. In Fig. 3, we show the posteriors for the dimensionless spin magnitudes $a_{1,2}$ : The bound on $a_{1}$ is about $20 \%$ more stringent for precessing EOBNR. This is true even if we account for the larger finite-sampling uncertainty in the precessing EOBNR quantiles (see Table I). The final spin presented in Table I and Fig. 1 was obtained, including the contribution from the in-plane spin components to the final spin [57]; previous publications [1,2] just use the contribution from the aligned components of the spins, which remains sufficient for the final mass computation. Just using the aligned components does not change the precessing EOBNR result but gives a precessing IMRPhenom result of $0.66_{-0.06}^{+0.04}$.

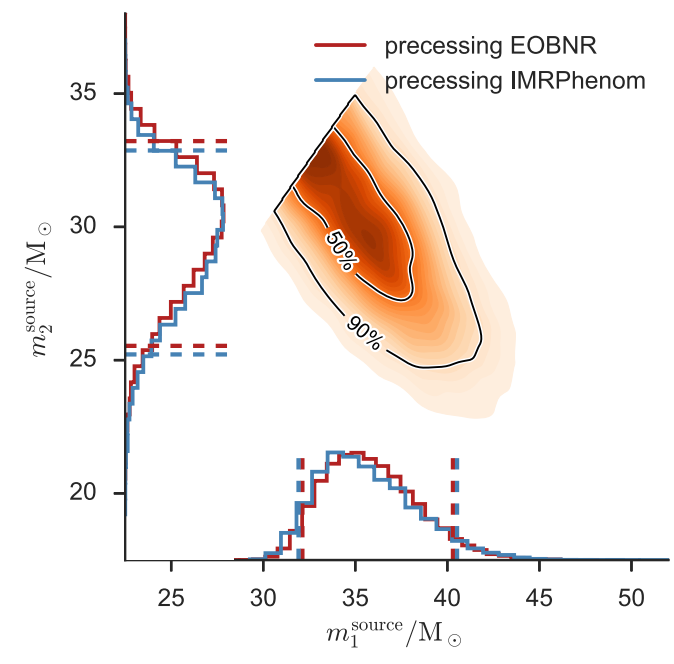

FIG. 2. Posterior probability densities for the source-frame component masses $m_{1}^{\text {source }}$ and $m_{2}^{\text {source }}$, where $m_{2}^{\text {source }} \leq m_{1}^{\text {source }}$. We show one-dimensional histograms for precessing EOBNR (red) and precessing IMRPhenom (blue); the dashed vertical lines mark the $90 \%$ credible intervals. The two-dimensional density plot shows $50 \%$ and $90 \%$ credible regions plotted over a colorcoded posterior density function.

Posterior histograms: Spin directions.-Figure 4 reproduces the disk plot of Ref. [2] for precessing EOBNR. In this plot, the three-dimensional histograms of the dimensionless spin vectors $S_{1,2} / m_{1,2}^{2}$ are projected onto a plane perpendicular to the orbital plane; the bins are designed so that each contains the same prior probability mass (i.e., histogramming the prior would result in a uniform shading). It is apparent that the data disfavor large spins aligned or antialigned with the orbital angular momentum, consistently with precessing IMRPhenom results. Because precessing EOBNR favors smaller values of the dimensionless spin magnitudes, the plot is darker towards its

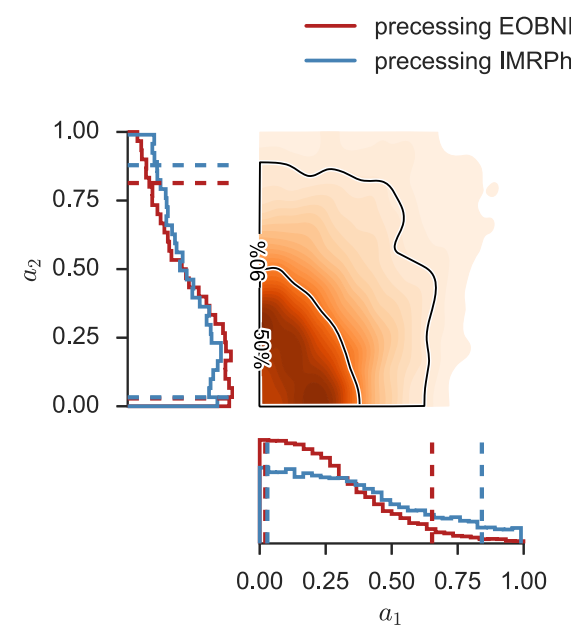

FIG. 3. Posterior probability densities for the dimensionless spin magnitudes. (See Fig. 2 for details.) 


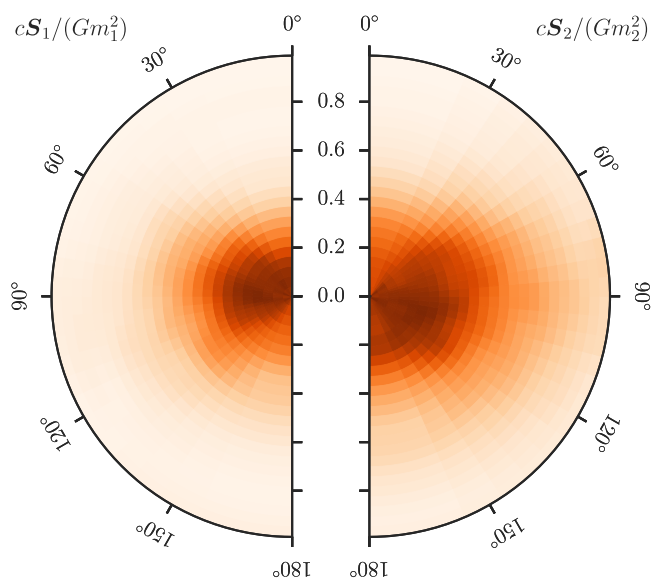

FIG. 4. Posterior probability density of $\mathrm{BH}$ spin directions, plotted as in Fig. 5 of Ref. [2].

center than its counterpart in Ref. [2]. In agreement with that paper, our analysis does not support strong statements on the orientation of the $\mathrm{BH}$ spins with respect to the orbital angular momentum. The spin opening angles (the tilts), defined by $\cos \left(\theta_{L S_{1,2}}\right)=\left(\boldsymbol{S}_{1,2} \cdot \hat{\boldsymbol{L}}_{\mathrm{N}}\right) /\left|\boldsymbol{S}_{1,2}\right|$, are distributed broadly. However, the KS test described at the end of Sec. III does indicate some deviation between priors and posteriors, with $p$-values much smaller than 0.05 for $\cos \left(\theta_{L S_{1}}\right)$ and $\cos \left(\theta_{L S_{2}}\right)$.

Posterior histograms: Effective spin parameters. - In Fig. 5, we show the posteriors of the effective spin combinations $\chi_{\text {eff }}[23,58-60]$ and $\chi_{\mathrm{p}}$ [44] defined by

$$
\begin{gathered}
\chi_{\text {eff }}=\frac{c}{G}\left(\frac{\boldsymbol{S}_{1}}{m_{1}}+\frac{\boldsymbol{S}_{2}}{m_{2}}\right) \cdot \frac{\hat{\boldsymbol{L}}_{\mathrm{N}}}{M}, \\
\chi_{\mathrm{p}}=\frac{c}{B_{1} G m_{1}^{2}} \max \left(B_{1} S_{1 \perp}, B_{2} S_{2 \perp}\right),
\end{gathered}
$$

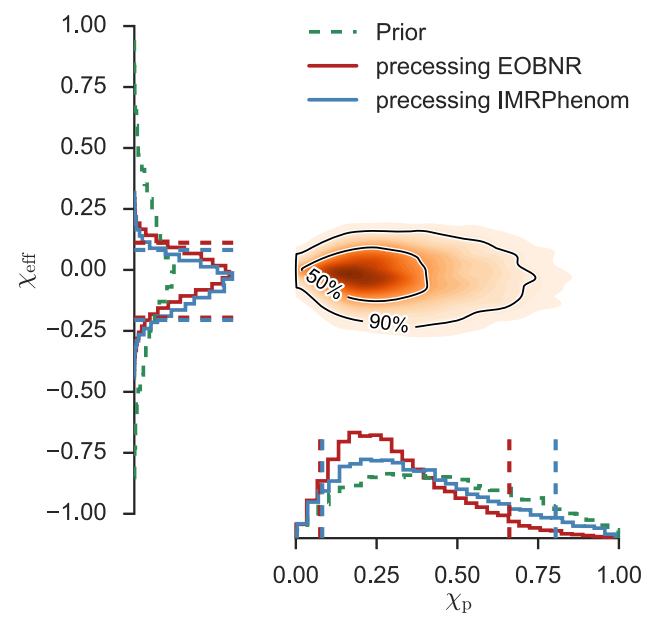

FIG. 5. Posterior probability densities of the effective spin and perpendicular effective spin. (See Fig. 2 for details.) where $S_{i \perp}$ is the component of the spin perpendicular to the orbital angular momentum $\boldsymbol{L}_{\mathrm{N}}, M$ is the total observed mass, $B_{1}=2+3 q / 2$ and $B_{2}=2+3 /(2 q)$, and $i=\{1,2\}$.

While $\chi_{\text {eff }}$ combines the projections of the $\mathrm{BH}$ spins onto the orbital angular momentum, $\chi_{\mathrm{p}}$ depends on their in-plane components and thus relates to precessional effects. Both models have credible intervals for $\chi_{\text {eff }}$ that contain the value 0 and deviate from the prior significantly. The data provide little information about precession but show a slightly stronger preference for lower values of $\chi_{\mathrm{p}}$ than expressed by our priors; the deviation is more pronounced for precessing EOBNR. The $90 \%$ credible intervals contain the value 0 and extend up to about 0.7 and 0.8 for precessing EOBNR and precessing IMRPhenom, respectively. Thus, precessing EOBNR provides a tighter upper bound.

Posterior histograms: Other spin angles.-To explore other possible differences between the two precessing models, we now consider spin parameters that were not reported in Ref. [2]. In particular, we compute posteriors for $\theta_{12}$, the opening angle between the spin vectors, and $\phi_{12}$, the opening angle between the in-plane projections of the spins. The prior on $\cos \theta_{12}$ is uniform in $[-1,1]$, while the prior on $\phi_{12}$ is uniform in $[0,2 \pi]$. We show these posteriors in Fig. 6. The $\theta_{12}$ posteriors deviate appreciably from the prior and are rather similar. By contrast, comparing the opening angle between spin projections onto the orbital plane, $\phi_{12}$, we find that the precessing EOBNR posterior deviates significantly from the prior (with $\mathrm{KS} p$-value $\in[0.0077,0.075])$, while the precessing IMRPhenom posterior does not (with $\mathrm{KS} p$-value $\in[0.30,0.60]$ ). This result is as it should be since in precessing IMRPhenom binaries with identical projection of the total spin on the orbital plane have identical waveforms. Although the KS $p$-values

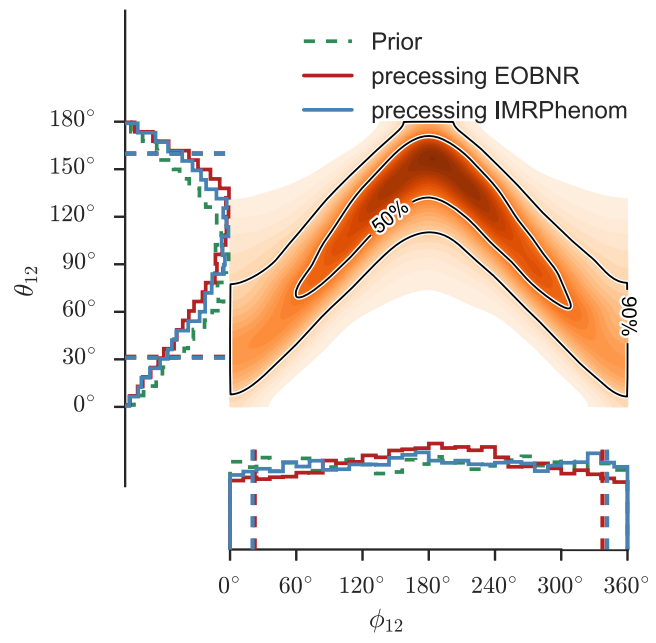

FIG. 6. Posterior probability densities of the opening angle between the two spins, $\theta_{12}$, and the angle between the in-plane projections of the spins, $\phi_{12}$. (See Fig. 2 for details.) 
suggest that the data provide information about $\theta_{12}$ and $\phi_{12}$ beyond the prior, we note that the $90 \%$ credible intervals for both of these parameters cover approximately $90 \%$ of their valid ranges and are indistinguishable for each waveform model.

Spin evolution.-All the source parameters discussed above are measured at a reference frequency of $20 \mathrm{~Hz}$. Exploiting the capability of precessing EOBNR of evolving the $\mathrm{BH}$ spin vectors in the time domain, we may address the question of estimating values for the spin parameters at the time of the merger. To do so, we randomly sample 1000 distinct configurations from the precessing EOBNR posteriors, and we evolve them to the maximum EOB orbital frequency (a proxy for the merger in the model). We then produce histograms of the evolved values of $\chi_{\text {eff }}$ and $\chi_{\mathrm{p}}$. We find little if any change between $20 \mathrm{~Hz}$ and the merger. Indeed, a KS test suggests that the original and evolved distributions are very consistent, with $p$-values close to 1 .

Comparison with numerical relativity.-The precessing EOBNR waveform model has been tested against NR waveforms using simulations from the SXS catalog $[17,18,36]$. We can provide a targeted cross-check on the accuracy of precessing EOBNR near GW150914 by performing parameter estimation runs on mock NR signals injected into LIGO data. This test is complementary to an ongoing study of the same nature, which, however, does not employ the precessing EOBNR model used in this paper. We use a new LAL infrastructure [61,62] to inject splineinterpolated and tapered NR waveforms into detector data; spins are defined with respect to the orbital angular momentum at a reference frequency of $20 \mathrm{~Hz}$. All higher harmonics of the GW signal are included up to the $l=8$ multipole. At the inclinations used in this study, the impact of modes with $l>2$ is small but merits further study; a detailed analysis will be presented in a forthcoming paper. We restrict this investigation to a NR waveform that was computed by the SXS Collaboration using the SpEC [63] code and is available in the public waveform catalogue [64] as SXS:BBH:0308. The intrinsic parameters of the NR waveform $q=0.81, a_{1}=0.34$, and $a_{2}=0.67$ are consistent with the results obtained in Ref. [2], and this waveform agrees well with the detector data.

We can freely choose the angle between the line of sight and the angular momentum of the binary for mock NR signals. Since there is some uncertainty in the binary's inclination, we perform one run near maximum a posteriori probability inclination, $\imath=2.856 \mathrm{rad}\left(163.6^{\circ}\right)$, and a second one at the upper bound of the $90 \%$ credible interval of the marginal probability density function (PDF) of the inclination, $\imath=1.2 \mathrm{rad}\left(68.8^{\circ}\right)$. In Fig. 7, we show the two GW polarizations for the NR waveform and the precessing EOBNR model. The spin magnitudes and the mass ratio were fixed to the NR values. The directions of spins are defined to be the same at the initial time: Tilt angles are $18.8^{\circ}, 149.4^{\circ}$, and the azimuthal angles, defined with respect to the initial separation vector, are $30.9^{\circ}, 38.7^{\circ}$ for the primary and secondary BHs, respectively. To quantify the agreement between those waveforms, we compute overlaps averaged over the GW polarization and source sky location, which takes into account the uncertainty in those parameters. The polarization-sky-averaged overlap for $\mathrm{MaP}$ inclination is 0.997 , and for $l=1.2 \mathrm{rad}\left(68.8^{\circ}\right)$, overlap is 0.993 .

We show results for the run with $\mathrm{MaP}$ inclination for the source-frame component masses and effective spins in the left and right panels of Fig. 8. The precessing EOBNR and precessing IMRPhenom models show good agreement in the masses and effective precession spin $\chi_{\mathrm{p}}$. The posterior PDFs obtained for the effective aligned spin $\chi_{\text {eff }}$ are slightly offset. All injected values are found within the 90\% credible regions. Results for the inclination chosen at the upper bound of the $90 \%$ credible interval of the marginal $\mathrm{PDF}$ of the inclination are qualitatively similar to the MaP results, except for the PDF of the effective precession spin, which peaks around $\chi_{\mathrm{p}} \sim 0.4$, noticeably above the injected value but still well inside the $90 \%$ credible interval.
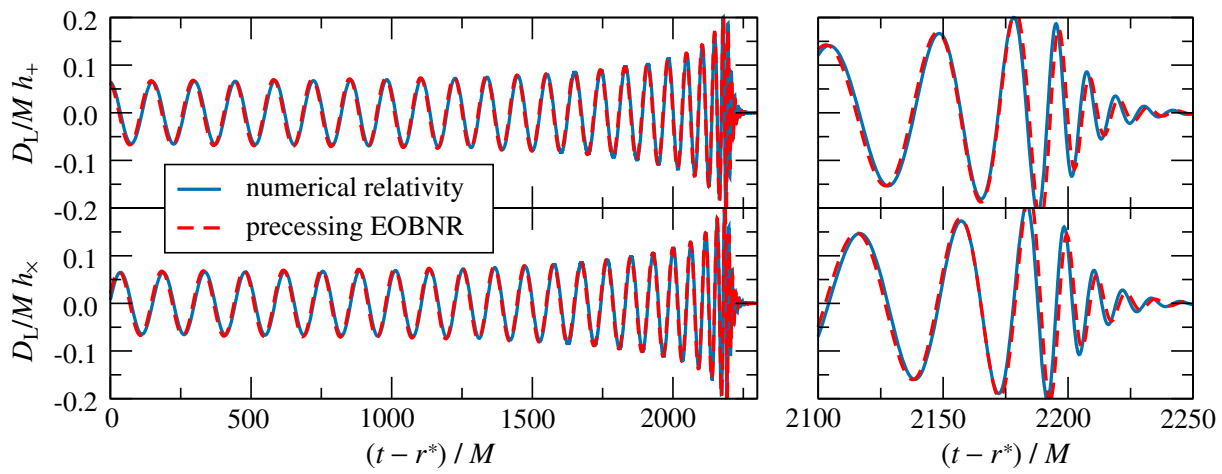

FIG. 7. A visual comparison of the precessing EOBNR model with NR GW polarizations computed by the SXS Collaboration at (approximately) the GW150914 MaP parameters. The intrinsic parameters of the NR waveform are $q=0.81, a_{1}=0.34, a_{2}=0.67$. The inclination is $\imath=2.856 \mathrm{rad}$. The alignment of the precessing EOBNR waveform is obtained from the sky- and polarizationaveraged overlap with the NR waveform. For more details on the alignment procedure, see Ref. [18]. 

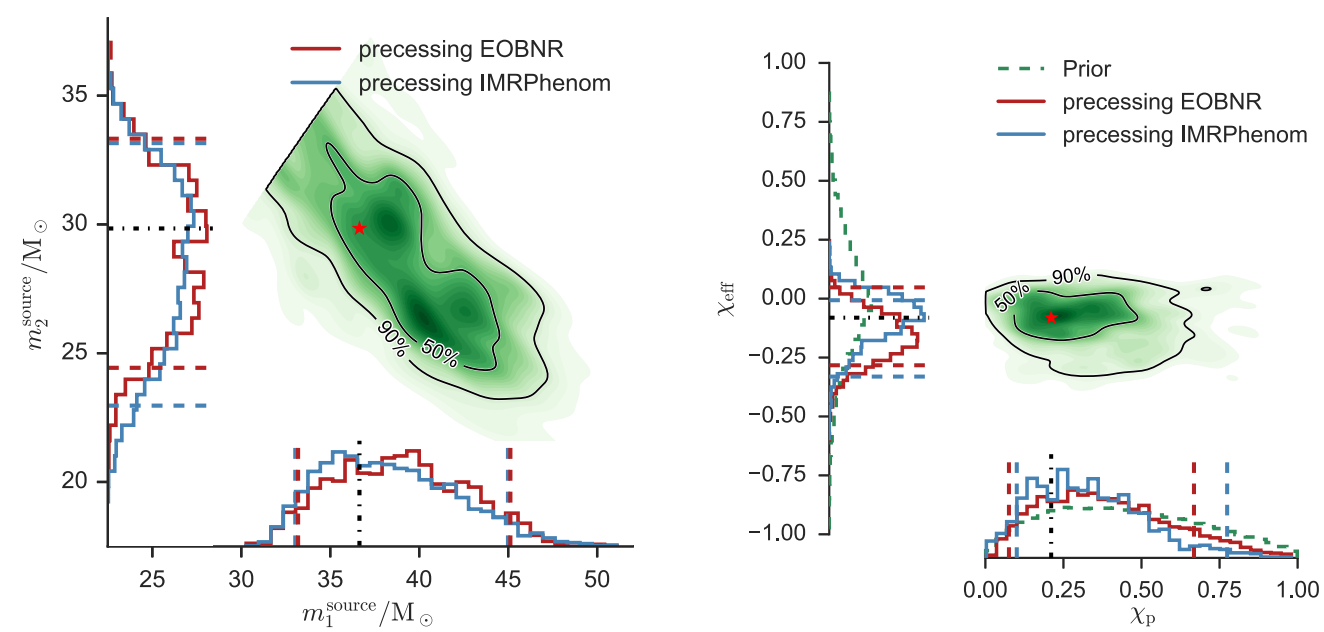

FIG. 8. Posterior probability densities for the source-frame component masses $m_{1}^{\text {source }}$ and $m_{2}^{\text {source }}$, where $m_{2}^{\text {source }} \leq m_{1}^{\text {source }}$ (left panel) and effective aligned $\chi_{\text {eff }}$ and effective precessing spins $\chi_{\mathrm{p}}$ (right panel) for an eventlike NR mock signal close to MaP parameters. In the one-dimensional marginalized distributions, we show the precessing EOBNR (red) and precessing IMRPhenom (blue) probability densities with dashed vertical lines marking $90 \%$ credible intervals. The two-dimensional plot shows the contours of the $50 \%$ and $90 \%$ credible regions of the precessing EOBNR over a color-coded posterior density function. The true parameter values are indicated by a red asterisk or black dot-dashed line.

\section{CONCLUSIONS}

We presented an updated analysis of GW150914 with mass estimates of $35_{-3}^{+5} \mathrm{M}_{\odot}$ and $30_{-4}^{+3} \mathrm{M}_{\odot}$, and we refined parameter estimates using a generalized, fully precessing waveform model that depends on all 15 independent parameters of a coalescing binary in circular orbit. We find this analysis to be broadly consistent with the results in Ref. [2]. By using the difference between two precessing waveform models as a proxy for systematic errors due to waveform uncertainty, we can compute a more accurate systematic error than what was possible in Ref. [2]. By looking at differences in 5\% and 95\% quantiles between different waveform models in Fig. 1, one can observe, on average, more consistent values when the two precessing models are compared. In addition, this analysis provides an estimate of the systematic error on precessing spin parameters such as the effective precessing spin $\chi_{\mathrm{p}}$ and the tilt angles $\left[\arccos \left(\hat{\boldsymbol{S}}_{1,2} \cdot \hat{\boldsymbol{L}}_{\mathrm{N}}\right)\right]$, which was not available in Ref. [2]. We have also carefully investigated uncertainties due to the finite numbers of samples used to recreate continuous posterior density functions, and we quantified their effects on quoted credible intervals. As in Ref. [2], the statistical error due to finite signal-to-noise ratio dominates the parameter-estimation error.

While we do recover a tighter limit on the spin magnitude of the most massive member of the binary that created GW150914 $(<0.65$ at $90 \%$ probability), the recovery of the spin parameters (magnitude and tilt angles) is too broad to hint at whether the black hole binary was formed via stellar binary interactions or dynamical capture [21]. This analysis on the first direct detection by LIGO will be applied to future detections [65], with the aim of getting the most accurate and most precise parameter estimate possible. In particular, binaries that have larger mass asymmetry, that are observed for a longer time, and that are more edge-on than GW150914 will display stronger spin-precession effects.

\section{ACKNOWLEDGMENTS}

The authors gratefully acknowledge the support of the United States National Science Foundation (NSF) for the construction and operation of the LIGO Laboratory and Advanced LIGO, as well as the Science and Technology Facilities Council (STFC) of the United Kingdom, the Max-Planck Society (MPS), and the State of Niedersachsen/Germany for support of the construction of Advanced LIGO and construction and operation of the GEO600 detector. Additional support for Advanced LIGO was provided by the Australian Research Council. The authors gratefully acknowledge the Italian Istituto Nazionale di Fisica Nucleare (INFN), the French Centre National de la Recherche Scientifique (CNRS) and the Foundation for Fundamental Research on Matter supported by the Netherlands Organisation for Scientific Research, for the construction and operation of the Virgo detector and the creation and support of the EGO consortium. The authors also gratefully acknowledge research support from these agencies, as well as by the Council of Scientific and Industrial Research of India; Department of Science and Technology, India; Science \& Engineering Research Board (SERB), India; Ministry of Human Resource Development, India; the Spanish Ministerio de Economía y Competitividad; the Conselleria d'Economia i Competitivitat and Conselleria d'Educació; Cultura i 
Universitats of the Govern de les Illes Balears; the National Science Centre of Poland; the European Commission; the Royal Society; the Scottish Funding Council; the Scottish Universities Physics Alliance; the Hungarian Scientific Research Fund (OTKA); the Lyon Institute of Origins (LIO); the National Research Foundation of Korea; Industry Canada and the Province of Ontario through the Ministry of Economic Development and Innovation; the Natural Science and Engineering Research Council Canada; Canadian Institute for Advanced Research; the Brazilian Ministry of Science, Technology, and Innovation; Russian Foundation for Basic Research; the Leverhulme Trust; the Research Corporation; Ministry of Science and Technology (MOST), Taiwan; and the Kavli Foundation.
The authors gratefully acknowledge the support of the NSF, STFC, MPS, INFN, CNRS, and the State of Niedersachsen/ Germany for provision of computational resources.

\section{APPENDIX: CREDIBLE INTERVALS FOR THE COMBINED POSTERIORS}

To compare directly with the results of Ref. [2], Fig. 9 presents the $90 \%$ credible intervals obtained with combined nonprecessing-EOBNR and precessing-IMRPhenom models, and with combined precessing-EOBNR precessingIMRPhenom models. As in Fig. 1, the darker bands visualize uncertainties due to the finite number of samples, as estimated with the Bayesian bootstrap.
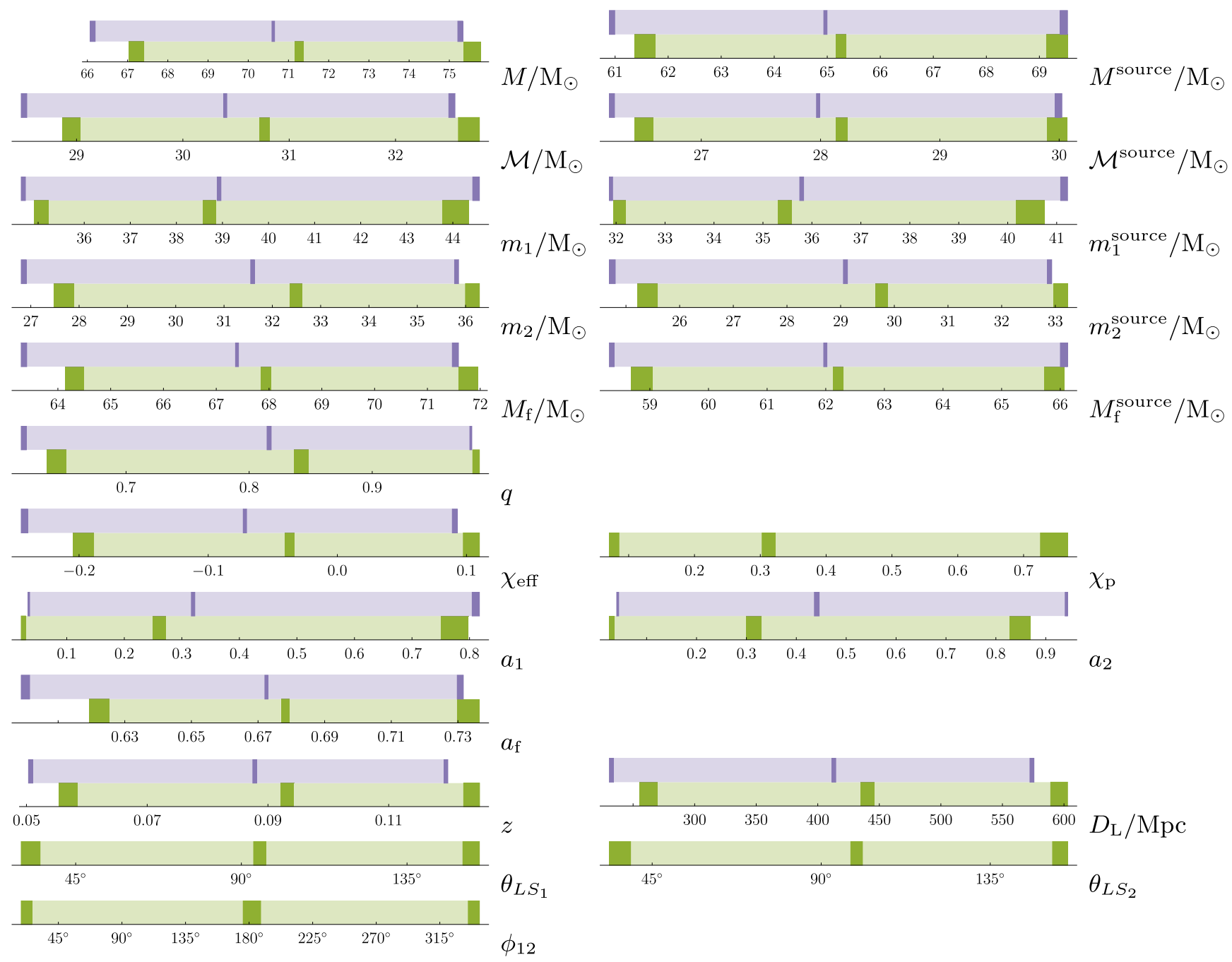

FIG. 9. Comparison of parameter estimates obtained by combining the nonprecessing-EOBNR and precessing-IMRPhenom models (as in Ref. [2]; light purple bars at the top) and by combining the precessing-EOBNR and precessing-IMRPhenom models (light green bars at the bottom). We show 90\% credible intervals for selected GW150914 source parameters. The darker intervals represent uncertainty estimates for the $5 \%, 50 \%$, and $95 \%$ quantiles (from left to right), as estimated by the Bayesian bootstrap. 
[1] B. P. Abbott et al. (LIGO Scientific Collaboration and Virgo Collaboration), Observation of Gravitational Waves from a Binary Black Hole Merger, Phys. Rev. Lett. 116, 061102 (2016).

[2] B. P. Abbott et al. (LIGO Scientific Collaboration and Virgo Collaboration), Properties of the Binary Black Hole Merger GW150914, Phys. Rev. Lett. 116, 241102 (2016).

[3] B. Brügmann, J. A. Gonzalez, M. Hannam, S. Husa, U. Sperhake, and W. Tichy, Calibration of Moving Puncture Simulations, Phys. Rev. D 77, 024027 (2008).

[4] R. O'Shaughnessy, L. London, J. Healy, and D. Shoemaker, Precession During Merger: Strong Polarization Changes Are Observationally Accessible Features of Strong-Field Gravity During Binary Black Hole Merger, Phys. Rev. D 87, 044038 (2013).

[5] M. A. Scheel, M. Giesler, D. A. Hemberger, G. Lovelace, K. Kuper, M. Boyle, B. Szilágyi, and L. E. Kidder, Improved Methods for Simulating Nearly Extremal Binary Black Holes, Classical Quantum Gravity 32, 105009 (2015).

[6] T. Chu, H. Fong, P. Kumar, H. P. Pfeiffer, M. Boyle, D. A. Hemberger, L. E. Kidder, M. A. Scheel, and B. Szilágyi, On the Accuracy and Precision of Numerical Waveforms: Effect of Waveform Extraction Methodology, arXiv:1512.06800.

[7] C. O. Lousto, J. Healy, and H. Nakano, Spin Flips in Generic Black Hole Binaries, Phys. Rev. D 93, 044031 (2016).

[8] B. Szilágyi, J. Blackman, A. Buonanno, A. Taracchini, H. P. Pfeiffer, M. A. Scheel, T. Chu, L. E. Kidder, and Y. Pan, Approaching the Post-Newtonian Regime with Numerical Relativity: A Compact-Object Binary Simulation Spanning 350 Gravitational-Wave Cycles, Phys. Rev. Lett. 115, 031102 (2015).

[9] P. Kumar, K. Barkett, S. Bhagwat, N. Afshari, D. A. Brown, G. Lovelace, M. A. Scheel, and B. Szilgyi, Accuracy and Precision of Gravitational-Wave Models of Inspiraling Neutron Star-Black Hole Binaries with Spin: Comparison with Matter-Free Numerical Relativity in the LowFrequency Regime, Phys. Rev. D 92, 102001 (2015).

[10] C. O. Lousto and J. Healy, Flip-Flopping Binary Black Holes, Phys. Rev. Lett. 114, 141101 (2015).

[11] B. P. Abbott et al. (Virgo Collaboration and LIGO Scientific Collaboration), Directly Comparing GW150914 with Numerical Solutions of Einstein's Equations for Binary Black Hole Coalescence, arXiv:1606.01262.

[12] J. Blackman, S. E. Field, C. R. Galley, B. Szilgyi, M. A. Scheel, M. Tiglio, and D. A. Hemberger, Fast and Accurate Prediction of Numerical Relativity Waveforms from Binary Black Hole Coalescences Using Surrogate Models, Phys. Rev. Lett. 115, 121102 (2015).

[13] A. Buonanno and T. Damour, Effective One-Body Approach to General Relativistic Two-Body Dynamics, Phys. Rev. D 59, 084006 (1999).

[14] A. Buonanno and T. Damour, Transition from Inspiral to Plunge in Binary Black Hole Coalescences, Phys. Rev. D 62, 064015 (2000).

[15] A. Taracchini et al., Effective-One-Body Model for BlackHole Binaries with Generic Mass Ratios and Spins, Phys. Rev. D 89, 061502 (2014).

[16] M. Hannam, P. Schmidt, A. Bohé, L. Haegel, S. Husa, F. Ohme, G. Pratten, and M. Pürrer, Simple Model of Complete
Precessing Black-Hole-Binary Gravitational Waveforms, Phys. Rev. Lett. 113, 151101 (2014).

[17] Y. Pan, A. Buonanno, A. Taracchini, L. E. Kidder, A. H. Mroué, H. P. Pfeiffer, M. A. Scheel, and B. Szilgyi, InspiralMerger-Ringdown Waveforms of Spinning, Precessing Black-Hole Binaries in the Effective-One-Body Formalism, Phys. Rev. D 89, 084006 (2014).

[18] S. Babak, A. Taracchini, and A. Buonanno (to be published).

[19] When generating a precessing $\mathrm{BBH}$ waveform with mass ratio, total mass, and spin magnitudes equal to the median values reported in the "Overall" column of Table I, starting from $20 \mathrm{~Hz}$, we find that precessing EOBNR is a factor of 450 (3) slower than precessing IMRPhenom (nonprecessing EOBNR).

[20] J. Aasi et al. (LIGO Collaboration and Virgo Collaboration), Parameter Estimation for Compact Binary Coalescence Signals with the First Generation Gravitational-Wave Detector Network, Phys. Rev. D 88, 062001 (2013).

[21] B. P. Abbott et al. (LIGO Scientific Collaboration and Virgo Collaboration), Astrophysical Implications of the Binary Black-Hole Merger GW150914, Astrophys. J. 818, L22 (2016).

[22] In the LAL, as well as in technical publications, the precessing EOBNR model that we use is called SEOBNRv3.

[23] T. Damour, Coalescence of Two Spinning Black Holes: An Effective One-Body Approach, Phys. Rev. D 64, 124013 (2001).

[24] A. Buonanno, Y. Chen, and T. Damour, Transition from Inspiral to Plunge in Precessing Binaries of Spinning Black Holes, Phys. Rev. D 74, 104005 (2006).

[25] T. Damour, P. Jaranowski, and G. Schaefer, Effective One Body Approach to the Dynamics of Two Spinning Black Holes with Next-to-Leading Order Spin-Orbit Coupling, Phys. Rev. D 78, 024009 (2008).

[26] E. Barausse and A. Buonanno, An Improved Effective-OneBody Hamiltonian for Spinning Black-Hole Binaries, Phys. Rev. D 81, 084024 (2010).

[27] E. Barausse and A. Buonanno, Extending the Effective-OneBody Hamiltonian of Black-Hole Binaries to Include Nextto-Next-to-Leading Spin-Orbit Couplings, Phys. Rev. D 84, 104027 (2011).

[28] T. Damour and A. Nagar, New Effective-One-Body Description of Coalescing Nonprecessing Spinning Black-Hole Binaries, Phys. Rev. D 90, 044018 (2014).

[29] L. Blanchet, Gravitational Radiation from Post-Newtonian Sources and Inspiralling Compact Binaries, Living Rev. Relativ. 17, 2 (2014).

[30] T. Damour and A. Nagar, Faithful Effective-One-Body Waveforms of Small-Mass-Ratio Coalescing Black-Hole Binaries, Phys. Rev. D 76, 064028 (2007).

[31] T. Damour, B. R. Iyer, and A. Nagar, Improved Resummation of Post-Newtonian Multipolar Waveforms from Circularized Compact Binaries, Phys. Rev. D 79, 064004 (2009).

[32] Y. Pan, A. Buonanno, R. Fujita, E. Racine, and H. Tagoshi, Post-Newtonian Factorized Multipolar Waveforms for Spinning, Non-precessing Black-Hole Binaries, Phys. Rev. D 83, 064003 (2011); Phys. Rev. D 87, 109901(E) (2013). 
[33] C. V. Vishveshwara, Scattering of Gravitational Radiation by a Schwarzschild Black-Hole, Nature (London) 227, 936 (1970).

[34] W. H. Press, Long Wave Trains of Gravitational Waves from a Vibrating Black Hole, Astrophys. J. 170, L105 (1971).

[35] S. Chandrasekhar and S. L. Detweiler, The Quasi-Normal Modes of the Schwarzschild Black Hole, Proc. R. Soc. A A344, 441 (1975).

[36] A. H. Mroué et al., Catalog of 174 Binary Black Hole Simulations for Gravitational Wave Astronomy, Phys. Rev. Lett. 111, 241104 (2013).

[37] E. Barausse, A. Buonanno, S. A. Hughes, G. Khanna, S.O'Sullivan, and Y. Pan, Modeling Multipolar Gravitational-Wave Emission from Small Mass-Ratio Mergers, Phys. Rev. D 85, 024046 (2012).

[38] A. Taracchini, A. Buonanno, G. Khanna, and S. A. Hughes, Small Mass Plunging into a Kerr Black Hole: Anatomy of the Inspiral-Merger-Ringdown Waveforms, Phys. Rev. D 90, 084025 (2014).

[39] A. Buonanno, Y.-b. Chen, Y. Pan, and M. Vallisneri, A Quasi-Physical Family of Gravity-Wave Templates for Precessing Binaries of Spinning Compact Objects: II. Application to Double-Spin Precessing Binaries, Phys. Rev. D 70, 104003 (2004); 74, 029902(E) (2006).

[40] M. Boyle, R. Owen, and H. P. Pfeiffer, A Geometric Approach to the Precession of Compact Binaries, Phys. Rev. D 84, 124011 (2011).

[41] P. Schmidt, M. Hannam, S. Husa, and P. Ajith, Tracking the Precession of Compact Binaries from Their GravitationalWave Signal, Phys. Rev. D 84, 024046 (2011).

[42] R. O'Shaughnessy, B. Vaishnav, J. Healy, Z. Meeks, and D. Shoemaker, Efficient Asymptotic Frame Selection for Binary Black Hole Spacetimes Using Asymptotic Radiation, Phys. Rev. D 84, 124002 (2011).

[43] P. Schmidt, M. Hannam, and S. Husa, Towards Models of Gravitational Waveforms from Generic Binaries: A Simple Approximate Mapping Between Precessing and Nonprecessing Inspiral Signals, Phys. Rev. D 86, 104063 (2012).

[44] P. Schmidt, F. Ohme, and M. Hannam, Towards Models of Gravitational Waveforms from Generic Binaries II: Modelling Precession Effects with a Single Effective Precession Parameter, Phys. Rev. D 91, 024043 (2015).

[45] In LAL, this precessing model is called IMRPhenomPv2.

[46] S. Khan, S. Husa, M. Hannam, F. Ohme, M. Pürrer, X. J. Forteza, and A. Bohé, Frequency-Domain Gravitational Waves from Nonprecessing Black-Hole Binaries. II. A Phenomenological Model for the Advanced Detector Era, Phys. Rev. D 93, 044007 (2016).

[47] However, $(2, \pm 1)$ modes in the inertial frame of the observer are generated by the change of frame.

[48] P. Kumar, T. Chu, H. Fong, H. P. Pfeiffer, M. Boyle, D. A. Hemberger, L. E. Kidder, M. A. Scheel, and B. Szilagyi, Accuracy of Binary Black Hole Waveform Models for Aligned-Spin Binaries, Phys. Rev. D 93, 104050 (2016).

[49] J. Veitch et al., Parameter Estimation for Compact Binaries with Ground-Based Gravitational-Wave Observations
Using the LALInference Software Library, Phys. Rev. D 91, 042003 (2015).

[50] By construction, the precessing IMRPhenom inertial-frame polarizations $h_{+, \times}$depend on the arrival time and phase exactly as they would in a model that includes only $(2, \pm 2)$ inertial-frame modes. Thus, although precessing IMRPhenom does include $(2, \pm 1)$ inertial-frame modes, the analytical marginalization that we just discussed is exact.

[51] T. Bayes and R. Price, An Essay Towards Solving a Problem in the Doctrine of Chances, Phil. Trans. R. Soc. London 53, 370 (1763).

[52] E. T. Jaynes, Probability Theory: The Logic of Science, edited by G. Larry Bretthorst (Cambridge University Press, Cambridge, 2003).

[53] C. Cutler and E. E. Flanagan, Gravitational Waves from Merging Compact Binaries: How Accurately Can One Extract the Binary's Parameters from the Inspiral Wave Form?, Phys. Rev. D 49, 2658 (1994).

[54] B. P. Abbott et al. (LIGO Scientific Collaboration), Calibration of the Advanced LIGO Detectors for the Discovery of the Binary Black-Hole Merger GW150914, https://dcc .ligo.org/LIGO-P1500248/public/main.

[55] D. B. Rubin, The Bayesian Bootstrap, Annali di statistica 9, 130 (1981).

[56] For $n$ samples, this involves generating 1000 realizations of weights according to the $(n-1)$-variate Dirichlet distribution.

[57] N. K. Johnson-McDaniel et al., Determining the Final Spin of a Binary Black Hole System Including In-Plane Spins: Method and Checks of Accuracy, Technical Report No. LIGO-T1600168 (LIGO Project, 2016).

[58] E. Racine, Analysis of Spin Precession in Binary Black Hole Systems Including Quadrupole-Monopole Interaction, Phys. Rev. D 78, 044021 (2008).

[59] P. Ajith et al., Inspiral-Merger-Ringdown Waveforms for Black-Hole Binaries with Non-precessing Spins, Phys. Rev. Lett. 106, 241101 (2011).

[60] L. Santamaría, F. Ohme, P. Ajith, B. Brügmann, N. Dorband, M. Hannam, S. Husa, P. Moesta, D. Pollney, C. Reisswig, E. L. Robinson, J. Seiler, and B. Krishnan, Matching Post-Newtonian and Numerical Relativity Waveforms: Systematic Errors and a New Phenomenological Model for Non-precessing Black Hole Binaries, Phys. Rev. D 82, 064016 (2010).

[61] P. Schmidt and I. Harry, Numerical Relativity Injection Infrastructure (unpublished).

[62] P. Schmidt and C. Galley, Reduced-Order Spline Interpolants of Numerical Relativity Waveforms (unpublished).

[63] See http://www.black-holes.org/SpEC.html.

[64] See http://www.black-holes.org/waveforms.

[65] B. P. Abbott et al. (LIGO Scientific Collaboration and Virgo Collaboration), The Rate of Binary Black Hole Mergers Inferred from Advanced LIGO Observations Surrounding GW150914, https://dcc.ligo.org/LIGO-P1500217/ public/main. 
B. P. Abbott, ${ }^{1}$ R. Abbott,,${ }^{1}$ T. D. Abbott, ${ }^{2}$ M. R. Abernathy, ${ }^{3}$ F. Acernese,${ }^{4,5}$ K. Ackley, ${ }^{6}$ C. Adams, ${ }^{7}$ T. Adams,${ }^{8}$ P. Addesso, ${ }^{9}$ R. X. Adhikari, ${ }^{1}$ V. B. Adya, ${ }^{10}$ C. Affeldt, ${ }^{10}$ M. Agathos, ${ }^{11}$ K. Agatsuma, ${ }^{11}$ N. Aggarwal, ${ }^{12}$ O. D. Aguiar, ${ }^{13}$ L. Aiello, ${ }^{14,15}$ A. Ain, ${ }^{16}$ P. Ajith, ${ }^{17}$ B. Allen, ${ }^{10,18,19}$ A. Allocca, ${ }^{20,21}$ P. A. Altin, ${ }^{22}$ S. B. Anderson, ${ }^{1}$ W. G. Anderson, ${ }^{18}$ K. Arai, ${ }^{1}$ M. C. Araya, ${ }^{1}$ C. C. Arceneaux,${ }^{23}$ J. S. Areeda, ${ }^{24}$ N. Arnaud,${ }^{25}$ K. G. Arun, ${ }^{26}$ S. Ascenzi,,${ }^{27,15}$ G. Ashton, ${ }^{28}$ M. Ast, ${ }^{29}$ S. M. Aston, ${ }^{7}$ P. Astone, ${ }^{30}$ P. Aufmuth, ${ }^{19}$ C. Aulbert, ${ }^{10}$ S. Babak,${ }^{31}$ P. Bacon,${ }^{32}$ M. K. M. Bader, ${ }^{11}$ P. T. Baker ${ }^{33}$ F. Baldaccini, ${ }^{34,35}$ G. Ballardin, ${ }^{36}$ S. W. Ballmer, ${ }^{37}$ J. C. Barayoga, ${ }^{1}$ S. E. Barclay, ${ }^{38}$ B. C. Barish, ${ }^{1}$ D. Barker, ${ }^{39}$ F. Barone, ${ }^{4,5}$ B. Barr, ${ }^{38}$ L. Barsotti, ${ }^{12}$ M. Barsuglia, ${ }^{32}$ D. Barta, ${ }^{40}$ J. Bartlett, ${ }^{39}$ I. Bartos,${ }^{41}$ R. Bassiri, ${ }^{42}$ A. Basti, ${ }^{20,21}$ J. C. Batch, ${ }^{39}$ C. Baune, ${ }^{10}$ V. Bavigadda, ${ }^{36}$ M. Bazzan, ${ }^{43,44}$ M. Bejger ${ }^{45}$ A. S. Bell, ${ }^{38}$ B. K. Berger, ${ }^{1}$ G. Bergmann, ${ }^{10}$ C. P. L. Berry, ${ }^{46}$ D. Bersanetti, ${ }^{47,48}$ A. Bertolini, ${ }^{11}$ J. Betzwieser, ${ }^{7}$ S. Bhagwat,${ }^{37}$ R. Bhandare, ${ }^{49}$ I. A. Bilenko, ${ }^{50}$ G. Billingsley, ${ }^{1}$ J. Birch, ${ }^{7}$ R. Birney, ${ }^{51}$ O. Birnholtz, ${ }^{10}$ S. Biscans, ${ }^{12}$ A. Bisht,${ }^{10,19}$ M. Bitossi, ${ }^{36}$ C. Biwer,${ }^{37}$ M. A. Bizouard,,${ }^{25}$ J. K. Blackburn, ${ }^{1}$ C. D. Blair, ${ }^{52}$ D. G. Blair, ${ }^{52}$ R. M. Blair, ${ }^{39}$ S. Bloemen, ${ }^{53}$ O. Bock, ${ }^{10}$ M. Boer, ${ }^{54}$ G. Bogaert,${ }^{54}$ C. Bogan, ${ }^{10}$ A. Bohe,${ }^{31}$ C. Bond,${ }^{46}$ F. Bondu, ${ }^{55}$ R. Bonnand, ${ }^{8}$ B. A. Boom, ${ }^{11}$ R. Bork, ${ }^{1}$ V. Boschi, ${ }^{20,21}$ S. Bose, ${ }^{56,16}$ Y. Bouffanais,${ }^{32}$ A. Bozzi, ${ }^{36}$ C. Bradaschia, ${ }^{21}$ P. R. Brady,${ }^{18}$ V. B. Braginsky, ${ }^{50,}$ M. Branchesi,${ }^{57,58}$ J. E. Brau,,${ }^{59}$ T. Briant,${ }^{60}$ A. Brillet,${ }^{54}$ M. Brinkmann, ${ }^{10}$ V. Brisson, ${ }^{25}$ P. Brockill, ${ }^{18}$ J. E. Broida, ${ }^{61}$ A. F. Brooks, ${ }^{1}$ D. A. Brown, ${ }^{37}$ D. D. Brown, ${ }^{46}$ N. M. Brown, ${ }^{12}$ S. Brunett, ${ }^{1}$ C. C. Buchanan, ${ }^{2}$ A. Buikema, ${ }^{12}$ T. Bulik, ${ }^{62}$ H. J. Bulten, ${ }^{63,11}$ A. Buonanno, ${ }^{31,64}$ D. Buskulic, ${ }^{8}$ C. Buy, ${ }^{32}$ R. L. Byer, ${ }^{42}$ M. Cabero, ${ }^{10}$ L. Cadonati, ${ }^{65}$ G. Cagnoli, ${ }^{66,67}$ C. Cahillane, ${ }^{1}$ J. Calderón Bustillo, ${ }^{65}$ T. Callister, ${ }^{1}$ E. Calloni, ${ }^{68,5}$ J. B. Camp, ${ }^{69}$

K. C. Cannon, ${ }^{70}$ J. Cao,${ }^{71}$ C. D. Capano, ${ }^{10}$ E. Capocasa, ${ }^{32}$ F. Carbognani, ${ }^{36}$ S. Caride, ${ }^{72}$ C. Casanueva Diaz, ${ }^{25}$

J. Casentini, ${ }^{27,15}$ S. Caudill, ${ }^{18}$ M. Cavaglià, ${ }^{23}$ F. Cavalier, ${ }^{25}$ R. Cavalieri, ${ }^{36}$ G. Cella, ${ }^{21}$ C. B. Cepeda, ${ }^{1}$ L. Cerboni Baiardi, ${ }^{57,58}$ G. Cerretani, ${ }^{20,21}$ E. Cesarini, ${ }^{27,15}$ S. J. Chamberlin, ${ }^{73}$ M. Chan, ${ }^{38}$ S. Chao,${ }^{74}$ P. Charlton, ${ }^{75}$ E. Chassande-Mottin, ${ }^{32}$ B. D. Cheeseboro, ${ }^{76}$ H. Y. Chen, ${ }^{77}$ Y. Chen, ${ }^{78}$ C. Cheng, ${ }^{74}$ A. Chincarini, ${ }^{48}$ A. Chiummo, ${ }^{36}$ H. S. Cho, ${ }^{79}$ M. Cho, ${ }^{64}$ J. H. Chow, ${ }^{22}$ N. Christensen, ${ }^{61}$ Q. Chu ${ }^{52}$ S. Chua, ${ }^{60}$ S. Chung, ${ }^{52}$ G. Ciani, ${ }^{6}$ F. Clara, ${ }^{39}$ J. A. Clark, ${ }^{65}$ F. Cleva, ${ }^{54}$ E. Coccia, ${ }^{27,14}$ P.-F. Cohadon, ${ }^{60}$ A. Colla, ${ }^{80,30}$ C. G. Collette, ${ }^{81}$ L. Cominsky, ${ }^{82}$ M. Constancio Jr., ${ }^{13}$ A. Conte, ${ }^{80,30}$ L. Conti, ${ }^{44}$ D. Cook, ${ }^{39}$ T. R. Corbitt, ${ }^{2}$ N. Cornish,${ }^{33}$ A. Corsi, ${ }^{72}$ S. Cortese, ${ }^{36}$ C. A. Costa, ${ }^{13}$ M. W. Coughlin, ${ }^{61}$ S. B. Coughlin, ${ }^{83}$ J.-P. Coulon, ${ }^{54}$ S. T. Countryman, ${ }^{41}$ P. Couvares, ${ }^{1}$ E. E. Cowan, ${ }^{65}$ D. M. Coward, ${ }^{52}$ M. J. Cowart, ${ }^{7}$ D. C. Coyne, ${ }^{1}$ R. Coyne, ${ }^{72}$ K. Craig, ${ }^{38}$ J. D. E. Creighton, ${ }^{18}$ J. Cripe, ${ }^{2}$ S. G. Crowder, ${ }^{84}$ A. Cumming, ${ }^{38}$ L. Cunningham, ${ }^{38}$ E. Cuoco, ${ }^{36}$ T. Dal Canton, ${ }^{10}$ S. L. Danilishin, ${ }^{38}$ S. D’Antonio, ${ }^{15}$ K. Danzmann, ${ }^{19,10}$ N. S. Darman, ${ }^{85}$ A. Dasgupta, ${ }^{86}$ C. F. Da Silva Costa, ${ }^{6}$ V. Dattilo, ${ }^{36}$

I. Dave, ${ }^{49}$ M. Davier, ${ }^{25}$ G. S. Davies, ${ }^{38}$ E. J. Daw,${ }^{87}$ R. Day,${ }^{36}$ S. De,${ }^{37}$ D. DeBra, ${ }^{42}$ G. Debreczeni,${ }^{40}$ J. Degallaix, ${ }^{66}$ M. De Laurentis, ${ }^{68,5}$ S. Deléglise, ${ }^{60}$ W. Del Pozzo, ${ }^{46}$ T. Denker, ${ }^{10}$ T. Dent,${ }^{10}$ V. Dergachev, ${ }^{1}$ R. De Rosa,${ }^{68,5}$ R. T. DeRosa, ${ }^{7}$

R. DeSalvo, ${ }^{9}$ R. C. Devine, ${ }^{76}$ S. Dhurandhar, ${ }^{16}$ M. C. Díaz,${ }^{88}$ L. Di Fiore ${ }^{5}$ M. Di Giovanni,${ }^{89,90}$ T. Di Girolamo, ${ }^{68,5}$ A. Di Lieto, ${ }^{20,21}$ S. Di Pace, ${ }^{80,30}$ I. Di Palma, ${ }^{31,80,30}$ A. Di Virgilio, ${ }^{21}$ V. Dolique,${ }^{66}$ F. Donovan, ${ }^{12}$ K. L. Dooley, ${ }^{23}$ S. Doravari, ${ }^{10}$ R. Douglas, ${ }^{38}$ T. P. Downes, ${ }^{18}$ M. Drago, ${ }^{10}$ R. W. P. Drever, ${ }^{1}$ J. C. Driggers, ${ }^{39}$ M. Ducrot, ${ }^{8}$ S. E. Dwyer, ${ }^{39}$ T. B. Edo ${ }^{87}$ M. C. Edwards, ${ }^{61}$ A. Effler, ${ }^{7}$ H.-B. Eggenstein, ${ }^{10}$ P. Ehrens, ${ }^{1}$ J. Eichholz, ${ }^{6,1}$ S. S. Eikenberry, ${ }^{6}$ W. Engels, ${ }^{78}$ R. C. Essick, ${ }^{12}$ Z. Etienne, ${ }^{76}$ T. Etzel, ${ }^{1}$ M. Evans,,${ }^{12}$ T. M. Evans, ${ }^{7}$ R. Everett,${ }^{73}$ M. Factourovich, ${ }^{41}$ V. Fafone,${ }^{27,15}$ H. Fair, ${ }^{37}$ S. Fairhurst, ${ }^{91}$ X. Fan, ${ }^{71}$ Q. Fang, ${ }^{52}$ S. Farinon, ${ }^{48}$ B. Farr, ${ }^{77}$ W. M. Farr, ${ }^{46}$ E. Fauchon-Jones,${ }^{91}$ M. Favata, ${ }^{92}$ M. Fays, ${ }^{91}$ H. Fehrmann, ${ }^{10}$ M. M. Fejer ${ }^{42}$ E. Fenyvesi ${ }^{93}$ I. Ferrante, ${ }^{20,21}$ E. C. Ferreira, ${ }^{13}$ F. Ferrini, ${ }^{36}$ F. Fidecaro,${ }^{20,21}$ I. Fiori, ${ }^{36}$ D. Fiorucci, ${ }^{32}$ R. P. Fisher, ${ }^{37}$ R. Flaminio, ${ }^{66,94}$ M. Fletcher, ${ }^{38}$ J.-D. Fournier, ${ }^{54}$ S. Frasca, ${ }^{80,30}$ F. Frasconi, ${ }^{21}$ Z. Frei, ${ }^{93}$ A. Freise ${ }^{46}$ R. Frey, ${ }^{59}$ V. Frey, ${ }^{25}$ P. Fritschel, ${ }^{12}$ V. V. Frolov, ${ }^{7}$ P. Fulda, ${ }^{6}$ M. Fyffe, ${ }^{7}$ H. A. G. Gabbard, ${ }^{23}$ S. Gaebel, ${ }^{46}$ J. R. Gair, ${ }^{95}$ L. Gammaitoni, ${ }^{34}$ S. G. Gaonkar, ${ }^{16}$ F. Garufi, ${ }^{68,5}$ G. Gaur, ${ }^{96,86}$ N. Gehrels, ${ }^{69}$ G. Gemme, ${ }^{48}$ P. Geng, ${ }^{88}$ E. Genin, ${ }^{36}$ A. Gennai, ${ }^{21}$ J. George ${ }^{49}$ L. Gergely, ${ }^{97}$ V. Germain, ${ }^{8}$ Abhirup Ghosh, ${ }^{17}$ Archisman Ghosh, ${ }^{17}$ S. Ghosh, ${ }^{53,11}$ J. A. Giaime, ${ }^{2,7}$ K. D. Giardina, ${ }^{7}$ A. Giazotto, ${ }^{21}$ K. Gill, ${ }^{98}$ A. Glaefke, ${ }^{38}$ E. Goetz, ${ }^{39}$ R. Goetz, ${ }^{6}$ L. Gondan, ${ }^{93}$ G. González, ${ }^{2}$ J. M. Gonzalez Castro, ${ }^{20,21}$ A. Gopakumar, ${ }^{99}$ N. A. Gordon, ${ }^{38}$ M. L. Gorodetsky, ${ }^{50}$ S. E. Gossan, ${ }^{1}$ M. Gosselin,${ }^{36}$ R. Gouaty, ${ }^{8}$ A. Grado, ${ }^{100,5}$ C. Graef, ${ }^{38}$ P. B. Graff, ${ }^{64}$ M. Granata, ${ }^{66}$ A. Grant, ${ }^{38}$ S. Gras, ${ }^{12}$ C. Gray, ${ }^{39}$ G. Greco, ${ }^{57,58}$ A. C. Green, ${ }^{46}$ P. Groot, ${ }^{53}$ H. Grote ${ }^{10}$ S. Grunewald, ${ }^{31}$ G. M. Guidi ${ }^{57,58}$ X. Guo, ${ }^{71}$ A. Gupta, ${ }^{16}$ M. K. Gupta, ${ }^{86}$ K. E. Gushwa, ${ }^{1}$ E. K. Gustafson, ${ }^{1}$ R. Gustafson, ${ }^{101}$ J. J. Hacker, ${ }^{24}$ B. R. Hall, ${ }^{56}$ E. D. Hall, ${ }^{1}$ G. Hammond ${ }^{38}$ M. Haney,${ }^{99}$ M. M. Hanke, ${ }^{10}$ J. Hanks, ${ }^{39}$ C. Hanna, ${ }^{73}$ M. D. Hannam, ${ }^{91}$ J. Hanson, ${ }^{7}$ T. Hardwick, ${ }^{2}$ J. Harms,${ }^{57,58}$ G. M. Harry, ${ }^{3}$ I. W. Harry, ${ }^{31}$ M. J. Hart, ${ }^{38}$ M. T. Hartman, ${ }^{6}$ C.-J. Haster, ${ }^{46}$ K. Haughian, ${ }^{38}$ J. Healy, ${ }^{102}$ A. Heidmann, ${ }^{60}$ M. C. Heintze, ${ }^{7}$ H. Heitmann, ${ }^{54}$ P. Hello, ${ }^{25}$ G. Hemming,${ }^{36}$ M. Hendry, ${ }^{38}$ I. S. Heng, ${ }^{38}$ J. Hennig, ${ }^{38}$ J. Henry, ${ }^{102}$ A. W. Heptonstall, ${ }^{1}$ M. Heurs, ${ }^{10,19}$ S. Hild,${ }^{38}$ D. Hoak, ${ }^{36}$ D. Hofman, ${ }^{66}$ K. Holt ${ }^{7}$ D. E. Holz,${ }^{77}$ P. Hopkins, ${ }^{91}$ J. Hough, ${ }^{38}$ E. A. Houston, ${ }^{38}$ E. J. Howell,${ }^{52}$ Y. M. Hu, ${ }^{10}$ S. Huang ${ }^{74}$ E. A. Huerta, ${ }^{103}$ D. Huet, ${ }^{25}$ B. Hughey, ${ }^{98}$ S. Husa, ${ }^{104}$ S. H. Huttner, ${ }^{38}$ T. Huynh-Dinh, ${ }^{7}$ N. Indik, ${ }^{10}$ D. R. Ingram, ${ }^{39}$ R. Inta, ${ }^{72}$ 
H. N. Isa, ${ }^{38}$ J.-M. Isac, ${ }^{60}$ M. Isi, ${ }^{1}$ T. Isogai, ${ }^{12}$ B. R. Iyer, ${ }^{17}$ K. Izumi,${ }^{39}$ T. Jacqmin, ${ }^{60}$ H. Jang, ${ }^{79}$ K. Jani, ${ }^{65}$ P. Jaranowski, ${ }^{105}$ S. Jawahar, ${ }^{106}$ L. Jian, ${ }^{52}$ F. Jiménez-Forteza, ${ }^{104}$ W. W. Johnson, ${ }^{2}$ N. K. Johnson-McDaniel, ${ }^{17}$ D. I. Jones, ${ }^{28}$ R. Jones, ${ }^{38}$ R. J. G. Jonker, ${ }^{11}$ L. Ju, ${ }^{52}$ Haris K, ${ }^{107}$ C. V. Kalaghatgi, ${ }^{91}$ V. Kalogera, ${ }^{83}$ S. Kandhasamy, ${ }^{23}$ G. Kang, ${ }^{79}$ J. B. Kanner, ${ }^{1}$ S. J. Kapadia, ${ }^{10}$ S. Karki, ${ }^{59}$ K. S. Karvinen, ${ }^{10}$ M. Kasprzack, ${ }^{36,2}$ E. Katsavounidis, ${ }^{12}$ W. Katzman, ${ }^{7}$ S. Kaufer, ${ }^{19}$ T. Kaur, ${ }^{52}$ K. Kawabe, ${ }^{39}$ F. Kéfélian, ${ }^{54}$ M. S. Kehl, ${ }^{108}$ D. Keitel, ${ }^{104}$ D. B. Kelley, ${ }^{37}$ W. Kells, ${ }^{1}$ R. Kennedy, ${ }^{87}$ J. S. Key,${ }^{88}$ F. Y. Khalili, ${ }^{50}$ I. Khan, ${ }^{14}$ S. Khan, ${ }^{91}$ Z. Khan, ${ }^{86}$ E. A. Khazanov, ${ }^{109}$ N. Kijbunchoo, ${ }^{39}$ Chi-Woong Kim, ${ }^{79}$ Chunglee Kim, ${ }^{79}$ J. Kim, ${ }^{110}$ K. Kim, ${ }^{111}$ N. Kim ${ }^{42}$ W. Kim, ${ }^{112}$ Y.-M. Kim, ${ }^{110}$ S. J. Kimbrell, ${ }^{65}$ E. J. King, ${ }^{112}$ P. J. King, ${ }^{39}$ J. S. Kissel, ${ }^{39}$ B. Klein, ${ }^{83}$ L. Kleybolte, ${ }^{29}$ S. Klimenko, ${ }^{6}$ S. M. Koehlenbeck, ${ }^{10}$ S. Koley, ${ }^{11}$ V. Kondrashov, ${ }^{1}$ A. Kontos, ${ }^{12}$ M. Korobko, ${ }^{29}$ W. Z. Korth, ${ }^{1}$ I. Kowalska ${ }^{62}$ D. B. Kozak, ${ }^{1}$ V. Kringel, ${ }^{10}$ A. Królak, ${ }^{113,114}$ C. Krueger, ${ }^{19}$ G. Kuehn, ${ }^{10}$ P. Kumar, ${ }^{108}$ R. Kumar, ${ }^{86}$ L. Kuo, ${ }^{74}$ A. Kutynia, ${ }^{113}$ B. D. Lackey, ${ }^{37}$ M. Landry, ${ }^{39}$ J. Lange, ${ }^{102}$ B. Lantz, ${ }^{42}$ P. D. Lasky, ${ }^{115}$ M. Laxen, ${ }^{7}$ A. Lazzarini, ${ }^{1}$ C. Lazzaro, ${ }^{44}$ P. Leaci, ${ }^{80,30}$ S. Leavey, ${ }^{38}$ E. O. Lebigot, ${ }^{32,71}$ C. H. Lee, ${ }^{110}$ H. K. Lee, ${ }^{111}$ H. M. Lee, ${ }^{116}$ K. Lee, ${ }^{38}$ A. Lenon, ${ }^{37}$ M. Leonardi, ${ }^{89,90}$ J. R. Leong, ${ }^{10}$ N. Leroy, ${ }^{25}$ N. Letendre, ${ }^{8}$ Y. Levin, ${ }^{115}$ J. B. Lewis, ${ }^{1}$ T. G. F. Li, ${ }^{117}$ A. Libson, ${ }^{12}$ T. B. Littenberg, ${ }^{118}$ N. A. Lockerbie, ${ }^{106}$ A. L. Lombardi, ${ }^{119}$ L. T. London, ${ }^{91}$ J. E. Lord, ${ }^{37}$ M. Lorenzini, ${ }^{14,15}$ V. Loriette, ${ }^{120}$ M. Lormand, ${ }^{7}$ G. Losurdo,${ }^{58}$ J. D. Lough, ${ }^{10,19}$ C. O. Lousto, ${ }^{102}$ G. Lovelace, ${ }^{24}$ H. Lück, ${ }^{19,10}$ A. P. Lundgren, ${ }^{10}$ R. Lynch, ${ }^{12}$ Y. Ma, ${ }^{52}$ B. Machenschalk, ${ }^{10}$ M. MacInnis,${ }^{12}$ D. M. Macleod, ${ }^{2}$ F. Magaña-Sandoval,${ }^{37}$ L. Magaña Zertuche, ${ }^{37}$ R. M. Magee ${ }^{56}$ E. Majorana, ${ }^{30}$ I. Maksimovic, ${ }^{120}$ V. Malvezzi, ${ }^{27,15}$ N. Man,${ }^{54}$ V. Mandic, ${ }^{84}$ V. Mangano, ${ }^{38}$ G. L. Mansell, ${ }^{22}$ M. Manske, ${ }^{18}$ M. Mantovani ${ }^{36}$ F. Marchesoni, ${ }^{121,35}$ F. Marion, ${ }^{8}$ S. Márka, ${ }^{41}$ Z. Márka, ${ }^{41}$ A. S. Markosyan, ${ }^{42}$ E. Maros, ${ }^{1}$ F. Martelli, ${ }^{57,58}$ L. Martellini, ${ }^{54}$ I. W. Martin, ${ }^{38}$ D. V. Martynov, ${ }^{12}$ J. N. Marx, ${ }^{1}$ K. Mason, ${ }^{12}$ A. Masserot, ${ }^{8}$ T. J. Massinger, ${ }^{37}$ M. Masso-Reid, ${ }^{38}$ S. Mastrogiovanni, ${ }^{80,30}$ F. Matichard, ${ }^{12}$ L. Matone,${ }^{41}$ N. Mavalvala, ${ }^{12}$ N. Mazumder, ${ }^{56}$ R. McCarthy, ${ }^{39}$ D. E. McClelland, ${ }^{22}$ S. McCormick, ${ }^{7}$ S. C. McGuire ${ }^{122}$ G. McIntyre, ${ }^{1}$ J. McIver, ${ }^{1}$ D. J. McManus,${ }^{22}$ T. McRae, ${ }^{22}$ S. T. McWilliams, ${ }^{76}$ D. Meacher, ${ }^{73}$ G. D. Meadors, ${ }^{31,10}$ J. Meidam, ${ }^{11}$ A. Melatos, ${ }^{85}$ G. Mendell, ${ }^{39}$ R. A. Mercer, ${ }^{18}$ E. L. Merilh, ${ }^{39}$ M. Merzougui, ${ }^{54}$ S. Meshkov, ${ }^{1}$ C. Messenger, ${ }^{38}$ C. Messick, ${ }^{73}$ R. Metzdorff, ${ }^{60}$ P. M. Meyers, ${ }^{84}$ F. Mezzani,${ }^{30,80}$ H. Miao, ${ }^{46}$ C. Michel,${ }^{66}$ H. Middleton, ${ }^{46}$ E. E. Mikhailov, ${ }^{123}$ L. Milano, ${ }^{68,5}$ A. L. Miller, ${ }^{6,80,30}$ A. Miller, ${ }^{83}$ B. B. Miller, ${ }^{83}$ J. Miller, ${ }^{12}$ M. Millhouse, ${ }^{33}$ Y. Minenkov, ${ }^{15}$ J. Ming, ${ }^{31}$ S. Mirshekari, ${ }^{124}$ C. Mishra, ${ }^{17}$ S. Mitra, ${ }^{16}$ V. P. Mitrofanov, ${ }^{50}$ G. Mitselmakher, ${ }^{6}$ R. Mittleman, ${ }^{12}$ A. Moggi, ${ }^{21}$ M. Mohan, ${ }^{36}$ S. R. P. Mohapatra, ${ }^{12}$ M. Montani, ${ }^{57,58}$ B. C. Moore,${ }^{92}$ C. J. Moore, ${ }^{125}$ D. Moraru, ${ }^{39}$ G. Moreno, ${ }^{39}$ S. R. Morriss,${ }^{88}$ K. Mossavi,${ }^{10}$ B. Mours, ${ }^{8}$ C. M. Mow-Lowry, ${ }^{46}$ G. Mueller ${ }^{6}$ A. W. Muir, ${ }^{91}$ Arunava Mukherjee, ${ }^{17}$ D. Mukherjee, ${ }^{18}$ S. Mukherjee, ${ }^{88}$ N. Mukund, ${ }^{16}$ A. Mullavey, J. Munch, ${ }^{112}$ D. J. Murphy, ${ }^{41}$ P. G. Murray, ${ }^{38}$ A. Mytidis, ${ }^{6}$ I. Nardecchia, ${ }^{27,15}$ L. Naticchioni, ${ }^{80,30}$ R. K. Nayak, ${ }^{126}$ K. Nedkova, ${ }^{119}$ G. Nelemans, ${ }^{53,11}$ T. J. N. Nelson, ${ }^{7}$ M. Neri, ${ }^{47,48}$ A. Neunzert, ${ }^{101}$ G. Newton, ${ }^{38}$ T. T. Nguyen, ${ }^{22}$ A. B. Nielsen, ${ }^{10}$ S. Nissanke, ${ }^{53,11}$ A. Nitz, ${ }^{10}$ F. Nocera ${ }^{36}$ D. Nolting, ${ }^{7}$ M. E. N. Normandin,${ }^{88}$ L. K. Nuttall, ${ }^{37}$ J. Oberling, ${ }^{39}$ E. Ochsner, ${ }^{18}$ J. O’Dell, ${ }^{127}$ E. Oelker, ${ }^{12}$ G. H. Ogin, ${ }^{128}$ J. J. Oh, ${ }^{129}$ S. H. Oh, ${ }^{129}$ F. Ohme, ${ }^{91}$ M. Oliver, ${ }^{104}$ P. Oppermann, ${ }^{10}$ Richard J. Oram, ${ }^{7}$ B. O'Reilly, ${ }^{7}$ R. O’Shaughnessy, ${ }^{102}$ D. J. Ottaway, ${ }^{12}$ H. Overmier, ${ }^{7}$ B. J. Owen, ${ }^{72}$ A. Pai, ${ }^{107}$ S. A. Pai, ${ }^{49}$ J. R. Palamos, ${ }^{59}$ O. Palashov, ${ }^{109}$ C. Palomba, ${ }^{30}$ A. Pal-Singh, ${ }^{29}$ H. Pan, ${ }^{74}$ C. Pankow, ${ }^{83}$ F. Pannarale, ${ }^{91}$ B. C. Pant, ${ }^{49}$ F. Paoletti, ${ }^{36,21}$ A. Paoli, ${ }^{36}$ M. A. Papa,${ }^{31,18,10}$ H. R. Paris,${ }^{42}$ W. Parker, ${ }^{7}$ D. Pascucci, ${ }^{38}$ A. Pasqualetti, ${ }^{36}$ R. Passaquieti, ${ }^{20,21}$ D. Passuello, ${ }^{21}$ B. Patricelli, ${ }^{20,21}$ Z. Patrick, ${ }^{42}$ B. L. Pearlstone, ${ }^{38}$ M. Pedraza, ${ }^{1}$ R. Pedurand,${ }^{66,130}$ L. Pekowsky, ${ }^{37}$ A. Pele, ${ }^{7}$ S. Penn, ${ }^{131}$ A. Perreca, ${ }^{1}$ L. M. Perri, ${ }^{83}$ H. P. Pfeiffer, ${ }^{108,31}$ M. Phelps, ${ }^{38}$ O. J. Piccinni, ${ }^{80,30}$ M. Pichot, ${ }^{54}$ F. Piergiovanni, ${ }^{57,58}$ V. Pierro, ${ }^{9}$ G. Pillant, ${ }^{36}$ L. Pinard ${ }^{66}$ I. M. Pinto, ${ }^{9}$ M. Pitkin, ${ }^{38}$ M. Poe, ${ }^{18}$ R. Poggiani, ${ }^{20,21}$ P. Popolizio, ${ }^{36}$ A. Post ${ }^{10}$ J. Powell, ${ }^{38}$ J. Prasad, ${ }^{16}$ V. Predoi,${ }^{91}$ T. Prestegard, ${ }^{84}$ L. R. Price, ${ }^{1}$ M. Prijatelj, ${ }^{10,36}$ M. Principe, ${ }^{9}$ S. Privitera, ${ }^{31}$ R. Prix,${ }^{10}$ G. A. Prodi,${ }^{89,90}$ L. Prokhorov, ${ }^{50}$ O. Puncken, ${ }^{10}$ M. Punturo, ${ }^{35}$ P. Puppo, ${ }^{30}$ M. Pürrer, ${ }^{31}$ H. Qi,${ }^{18}$ J. Qin, ${ }^{52}$ S. Qiu, ${ }^{115}$ V. Quetschke, ${ }^{88}$ E. A. Quintero, ${ }^{1}$ R. Quitzow-James, ${ }^{59}$ F. J. Raab, ${ }^{39}$ D. S. Rabeling, ${ }^{22}$ H. Radkins, ${ }^{39}$ P. Raffai, ${ }^{93}$ S. Raja ${ }^{49}$ C. Rajan, ${ }^{49}$ M. Rakhmanov, ${ }^{88}$ P. Rapagnani, ${ }^{80,30}$ V. Raymond, ${ }^{31}$ M. Razzano, ${ }^{20,21}$ V. Re, ${ }^{27}$ J. Read, ${ }^{24}$ C. M. Reed, ${ }^{39}$ T. Regimbau, ${ }^{54}$ L. Rei, ${ }^{48}$ S. Reid, ${ }^{51}$ D. H. Reitze, ${ }^{1,6}$ H. Rew, ${ }^{123}$ S. D. Reyes,${ }^{37}$ F. Ricci, ${ }^{80,30}$ K. Riles ${ }^{101}$ M. Rizzo, ${ }^{102}$ N. A. Robertson, ${ }^{1,38}$ R. Robie, ${ }^{38}$ F. Robinet, ${ }^{25}$ A. Rocchi, ${ }^{15}$ L. Rolland, ${ }^{8}$ J. G. Rollins, ${ }^{1}$ V. J. Roma, ${ }^{59}$ R. Romano, ${ }^{4,5}$ G. Romanov, ${ }^{123}$ J. H. Romie, ${ }^{7}$ D. Rosińska, ${ }^{132,45}$ S. Rowan, ${ }^{38}$ A. Rüdiger, ${ }^{10}$ P. Ruggi,${ }^{36}$ K. Ryan, ${ }^{39}$ S. Sachdev, ${ }^{1}$ T. Sadecki, ${ }^{39}$ L. Sadeghian, ${ }^{18}$ M. Sakellariadou, ${ }^{133}$ L. Salconi, ${ }^{36}$ M. Saleem, ${ }^{107}$ F. Salemi, ${ }^{10}$ A. Samajdar, ${ }^{126}$ L. Sammut, ${ }^{115}$ E. J. Sanchez, ${ }^{1}$ V. Sandberg, ${ }^{39}$ B. Sandeen, ${ }^{83}$ J. R. Sanders, ${ }^{37}$ B. Sassolas, ${ }^{66}$ B. S. Sathyaprakash, ${ }^{91}$ P. R. Saulson, ${ }^{37}$ O. E. S. Sauter, ${ }^{101}$ R. L. Savage ${ }^{39}$ A. Sawadsky, ${ }^{19}$ P. Schale, ${ }^{59}$ R. Schilling, ${ }^{10, \dagger}$ J. Schmidt, ${ }^{10}$ P. Schmidt, ${ }^{1,78}$ R. Schnabel, ${ }^{29}$

R. M. S. Schofield, ${ }^{59}$ A. Schönbeck, ${ }^{29}$ E. Schreiber, ${ }^{10}$ D. Schuette, ${ }^{10,19}$ B. F. Schutz, ${ }^{91,31}$ J. Scott, ${ }^{38}$ S. M. Scott, ${ }^{22}$ D. Sellers, ${ }^{7}$ A. S. Sengupta, ${ }^{96}$ D. Sentenac, ${ }^{36}$ V. Sequino, ${ }^{27,15}$ A. Sergeev, ${ }^{109}$ Y. Setyawati, ${ }^{53,11}$ D. A. Shaddock, ${ }^{22}$ T. Shaffer, ${ }^{39}$ 
M. S. Shahriar, ${ }^{83}$ M. Shaltev, ${ }^{10}$ B. Shapiro, ${ }^{42}$ P. Shawhan, ${ }^{64}$ A. Sheperd, ${ }^{18}$ D. H. Shoemaker, ${ }^{12}$ D. M. Shoemaker, ${ }^{65}$ K. Siellez, ${ }^{65}$ X. Siemens, ${ }^{18}$ M. Sieniawska, ${ }^{45}$ D. Sigg, ${ }^{39}$ A. D. Silva, ${ }^{13}$ A. Singer, ${ }^{1}$ L. P. Singer, ${ }^{69}$ A. Singh, ${ }^{31,10,19}$ R. Singh, ${ }^{2}$ A. Singhal, ${ }^{14}$ A. M. Sintes, ${ }^{104}$ B. J. J. Slagmolen, ${ }^{22}$ J. R. Smith, ${ }^{24}$ N. D. Smith, ${ }^{1}$ R. J. E. Smith, ${ }^{1}$ E. J. Son, ${ }^{129}$ B. Sorazu, ${ }^{38}$ F. Sorrentino, ${ }^{48}$ T. Souradeep, ${ }^{16}$ A. K. Srivastava, ${ }^{86}$ A. Staley, ${ }^{41}$ M. Steinke, ${ }^{10}$ J. Steinlechner, ${ }^{38}$ S. Steinlechner, ${ }^{38}$ D. Steinmeyer, ${ }^{10,19}$ B. C. Stephens, ${ }^{18}$ S. P. Stevenson, ${ }^{46}$ R. Stone, ${ }^{88}$ K. A. Strain, ${ }^{38}$ N. Straniero, ${ }^{66}$ G. Stratta, ${ }^{57,58}$ N. A. Strauss, ${ }^{61}$ S. Strigin, ${ }^{50}$ R. Sturani, ${ }^{124}$ A. L. Stuver, ${ }^{7}$ T. Z. Summerscales, ${ }^{134}$ L. Sun, ${ }^{85}$ S. Sunil, ${ }^{86}$ P. J. Sutton, ${ }^{91}$ B. L. Swinkels, ${ }^{36}$ M. J. Szczepańczyk, ${ }^{98}$ M. Tacca, ${ }^{32}$ D. Talukder, ${ }^{59}$ D. B. Tanner, ${ }^{6}$ M. Tápai, ${ }^{97}$ S. P. Tarabrin, ${ }^{10}$ A. Taracchini, ${ }^{31}$ R. Taylor, ${ }^{1}$ T. Theeg, ${ }^{10}$ M. P. Thirugnanasambandam, ${ }^{1}$ E. G. Thomas, ${ }^{46}$ M. Thomas, ${ }^{7}$ P. Thomas, ${ }^{39}$ K. A. Thorne, ${ }^{7}$ K. S. Thorne, ${ }^{78}$ E. Thrane, ${ }^{115}$ S. Tiwari, ${ }^{14,90}$ V. Tiwari, ${ }^{91}$ K. V. Tokmakov, ${ }^{106}$ K. Toland, ${ }^{38}$ C. Tomlinson, ${ }^{87}$ M. Tonelli, ${ }^{20,21}$ Z. Tornasi, ${ }^{38}$ C. V. Torres, ${ }^{88, \dagger}$ C. I. Torrie, ${ }^{1}$ D. Töyrä, ${ }^{46}$ F. Travasso, ${ }^{34,35}$ G. Traylor, ${ }^{7}$ D. Trifirò, ${ }^{23}$ M. C. Tringali, ${ }^{89,90}$ L. Trozzo, ${ }^{135,21}$ M. Tse, ${ }^{12}$ M. Turconi, ${ }^{54}$ D. Tuyenbayev, ${ }^{88}$ D. Ugolini, ${ }^{136}$ C. S. Unnikrishnan, ${ }^{99}$ A. L. Urban, ${ }^{18}$ S. A. Usman, ${ }^{37}$ H. Vahlbruch, ${ }^{19}$ G. Vajente, ${ }^{1}$ G. Valdes, ${ }^{88}$ M. Vallisneri, ${ }^{78}$ N. van Bakel, ${ }^{11}$ M. van Beuzekom, ${ }^{11}$ J. F. J. van den Brand, ${ }^{63,11}$ C. Van Den Broeck, ${ }^{11}$ D. C. Vander-Hyde, ${ }^{37}$ L. van der Schaaf, ${ }^{11}$ M. V. van der Sluys, ${ }^{53}$ J. V. van Heijningen, ${ }^{11}$ A. Vano-Vinuales, ${ }^{91}$ A. A. van Veggel, ${ }^{38}$ M. Vardaro, ${ }^{43,44}$ S. Vass, ${ }^{1}$ M. Vasúth, ${ }^{40}$ R. Vaulin, ${ }^{12}$ A. Vecchio, ${ }^{46}$ G. Vedovato, ${ }^{44}$ J. Veitch, ${ }^{46}$ P. J. Veitch, ${ }^{112}$ K. Venkateswara, ${ }^{137}$ D. Verkindt, ${ }^{8}$ F. Vetrano, ${ }^{57,58}$ A. Viceré, ${ }^{57,58}$ S. Vinciguerra, ${ }^{46}$ D. J. Vine, ${ }^{51}$ J.-Y. Vinet, ${ }^{54}$ S. Vitale, ${ }^{12}$ T. Vo, ${ }^{37}$ H. Vocca, ${ }^{34,35}$ C. Vorvick, ${ }^{39}$ D. V. Voss, ${ }^{6}$ W. D. Vousden, ${ }^{46}$ S. P. Vyatchanin, ${ }^{50}$ A. R. Wade, ${ }^{22}$ L. E. Wade, ${ }^{138}$ M. Wade, ${ }^{138}$ M. Walker, ${ }^{2}$ L. Wallace, ${ }^{1}$ S. Walsh, ${ }^{31,10}$ G. Wang, ${ }^{14,58}$ H. Wang, ${ }^{46}$ M. Wang, ${ }^{46}$ X. Wang, ${ }^{71}$ Y. Wang, ${ }^{52}$ R. L. Ward, ${ }^{22}$ J. Warner, ${ }^{39}$ M. Was, ${ }^{8}$ B. Weaver, ${ }^{39}$ L.-W. Wei, ${ }^{54}$ M. Weinert, ${ }^{10}$ A. J. Weinstein, ${ }^{1}$ R. Weiss, ${ }^{12}$ L. Wen, ${ }^{52}$ P. Weßels, ${ }^{10}$ T. Westphal, ${ }^{10}$ K. Wette, ${ }^{10}$ J. T. Whelan, ${ }^{102}$ B. F. Whiting, ${ }^{6}$ R. D. Williams, ${ }^{1}$ A. R. Williamson, ${ }^{91}$ J. L. Willis, ${ }^{139}$ B. Willke, ${ }^{19,10}$ M. H. Wimmer, ${ }^{10,19}$ W. Winkler, ${ }^{10}$ C. C. Wipf, ${ }^{1}$ H. Wittel, ${ }^{10,19}$ G. Woan, ${ }^{38}$ J. Woehler, ${ }^{10}$ J. Worden, ${ }^{39}$ J. L. Wright, ${ }^{38}$ D. S. Wu, ${ }^{10}$ G. Wu, ${ }^{7}$ J. Yablon, ${ }^{83}$ W. Yam, ${ }^{12}$ H. Yamamoto, ${ }^{1}$ C. C. Yancey, ${ }^{64}$ H. Yu, ${ }^{12}$ M. Yvert, ${ }^{8}$ A. Zadrożny, ${ }^{13}$ L. Zangrando, ${ }^{44}$ M. Zanolin, ${ }^{98}$ J.-P. Zendri, ${ }^{44}$ M. Zevin, ${ }^{83}$ L. Zhang, ${ }^{1}$ M. Zhang, ${ }^{123}$ Y. Zhang, ${ }^{102}$ C. Zhao, ${ }^{52}$ M. Zhou, ${ }^{83}$ Z. Zhou, ${ }^{83}$ X. J. Zhu, ${ }^{52}$ M. E. Zucker, ${ }^{1,12}$ S. E. Zuraw, ${ }^{119}$ J. Zweizig, ${ }^{1}$ M. Boyle, ${ }^{140}$ B. Brügmann, ${ }^{141}$ M. Campanelli, ${ }^{102}$ T. Chu, ${ }^{140}$ M. Clark, ${ }^{65}$ R. Haas, ${ }^{31}$ D. Hemberger, ${ }^{78}$ I. Hinder, ${ }^{31}$ L. E. Kidder, ${ }^{140}$ M. Kinsey, ${ }^{65}$ P. Laguna, ${ }^{65}$ S. Ossokine, ${ }^{31}$ Y. Pan, ${ }^{64}$ C. Röver, ${ }^{10}$ M. Scheel, ${ }^{78}$ B. Szilagyi, ${ }^{78,142}$ S. Teukolsky, ${ }^{140}$ and Y. Zlochower ${ }^{102}$

(LIGO Scientific Collaboration and Virgo Collaboration)

${ }^{1}$ LIGO, California Institute of Technology, Pasadena, California 91125, USA

${ }^{2}$ Louisiana State University, Baton Rouge, Louisiana 70803, USA

${ }^{3}$ American University, Washington, D.C. 20016, USA

${ }^{4}$ Università di Salerno, Fisciano, I-84084 Salerno, Italy

${ }^{5}$ INFN, Sezione di Napoli, Complesso Universitario di Monte S.Angelo, I-80126 Napoli, Italy

${ }^{6}$ University of Florida, Gainesville, Florida 32611, USA

${ }^{7}$ LIGO Livingston Observatory, Livingston, Louisiana 70754, USA

${ }^{8}$ Laboratoire d'Annecy-le-Vieux de Physique des Particules (LAPP), Université Savoie Mont Blanc, CNRS/IN2P3, F-74941 Annecy-le-Vieux, France

${ }^{9}$ University of Sannio at Benevento,

I-82100 Benevento, Italy and INFN, Sezione di Napoli, I-80100 Napoli, Italy

${ }^{10}$ Albert-Einstein-Institut, Max-Planck-Institut für Gravitationsphysik, D-30167 Hannover, Germany

${ }^{11}$ Nikhef, Science Park, 1098 XG Amsterdam, The Netherlands

${ }^{12}$ LIGO, Massachusetts Institute of Technology, Cambridge, Massachusetts 02139, USA

${ }^{13}$ Instituto Nacional de Pesquisas Espaciais, 12227-010 São José dos Campos, São Paulo, Brazil

${ }^{14}$ INFN, Gran Sasso Science Institute, I-67100L'Aquila, Italy

${ }^{15}$ INFN, Sezione di Roma Tor Vergata, I-00133 Roma, Italy

${ }^{16}$ Inter-University Centre for Astronomy and Astrophysics, Pune 411007, India

${ }^{17}$ International Centre for Theoretical Sciences, Tata Institute of Fundamental Research, Bangalore 560012, India

${ }^{18}$ University of Wisconsin-Milwaukee, Milwaukee, Wisconsin 53201, USA

${ }^{19}$ Leibniz Universität Hannover, D-30167 Hannover, Germany

${ }^{20}$ Università di Pisa, I-56127 Pisa, Italy

${ }^{21}$ INFN, Sezione di Pisa, I-56127 Pisa, Italy 


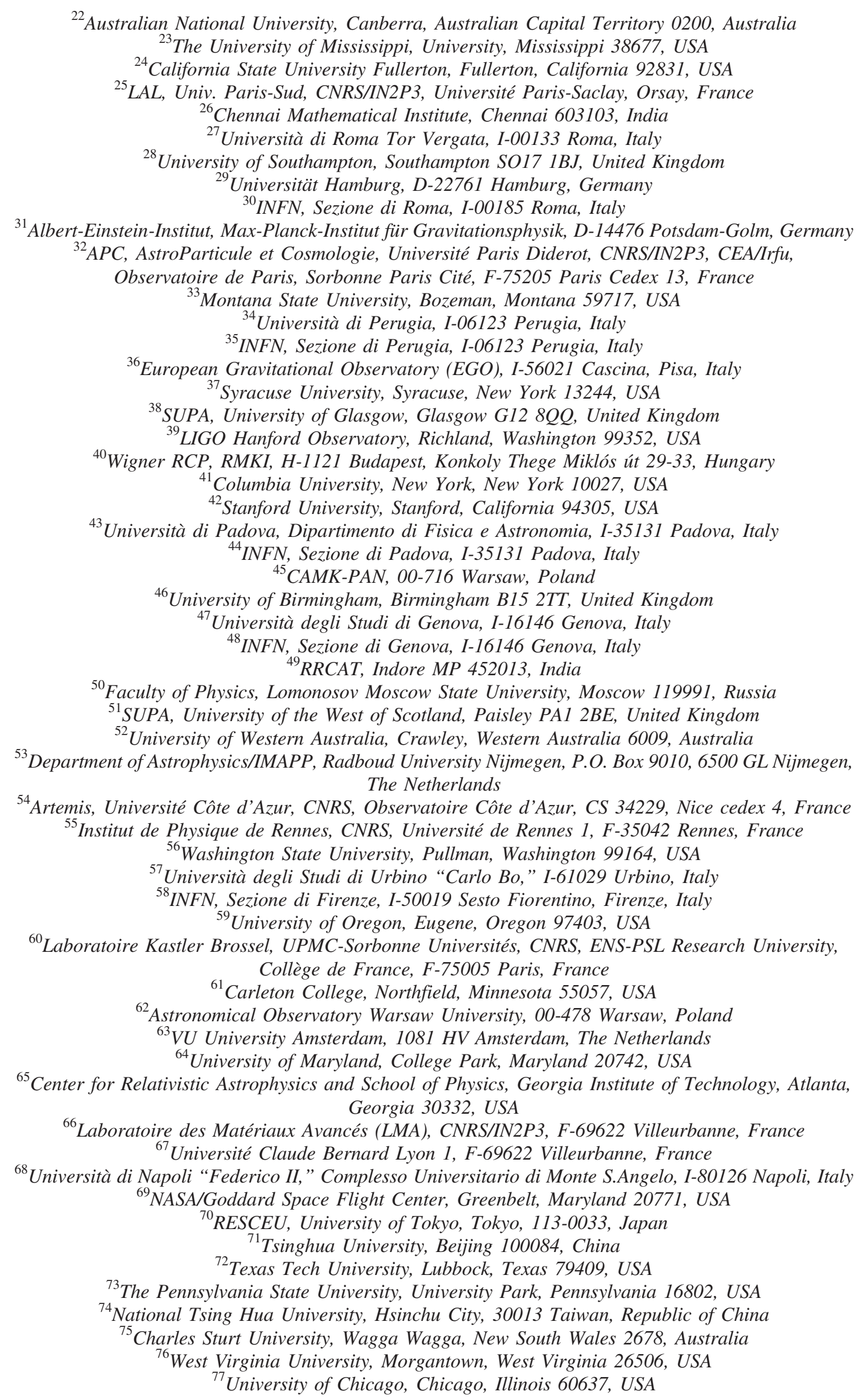




\author{
${ }^{78}$ Caltech CaRT, Pasadena, California 91125, USA \\ ${ }^{79}$ Korea Institute of Science and Technology Information, Daejeon 305-806, Korea \\ ${ }^{80}$ Università di Roma "La Sapienza”, I-00185 Roma, Italy \\ ${ }^{81}$ University of Brussels, Brussels 1050, Belgium \\ ${ }^{82}$ Sonoma State University, Rohnert Park, California 94928, USA \\ ${ }^{83}$ Center for Interdisciplinary Exploration \& Research in Astrophysics (CIERA), Northwestern University, \\ Evanston, Illinois 60208, USA \\ ${ }^{84}$ University of Minnesota, Minneapolis, Minnesota 55455, USA \\ ${ }^{85}$ The University of Melbourne, Parkville, Victoria 3010, Australia \\ ${ }^{86}$ Institute for Plasma Research, Bhat, Gandhinagar 382428, India \\ ${ }^{87}$ The University of Sheffield, Sheffield S10 2TN, United Kingdom \\ ${ }^{88}$ The University of Texas Rio Grande Valley, Brownsville, Texas 78520, USA \\ ${ }^{89}$ Università di Trento, Dipartimento di Fisica, I-38123 Povo, Trento, Italy \\ ${ }^{90}$ INFN, Trento Institute for Fundamental Physics and Applications, I-38123 Povo, Trento, Italy \\ ${ }^{91}$ Cardiff University, Cardiff CF24 3AA, United Kingdom \\ ${ }^{92}$ Montclair State University, Montclair, New Jersey 07043, USA \\ ${ }^{93}$ MTA Eötvös University, "Lendulet” Astrophysics Research Group, Budapest 1117, Hungary \\ ${ }^{94}$ National Astronomical Observatory of Japan, 2-21-1 Osawa, Mitaka, Tokyo 181-8588, Japan \\ ${ }^{95}$ School of Mathematics, University of Edinburgh, Edinburgh EH9 3FD, United Kingdom \\ ${ }^{96}$ Indian Institute of Technology, Gandhinagar, Ahmedabad, Gujarat 382424, India \\ ${ }^{97}$ University of Szeged, Dóm tér 9, Szeged 6720, Hungary \\ ${ }^{98}$ Embry-Riddle Aeronautical University, Prescott, Arizona 86301, USA \\ ${ }^{99}$ Tata Institute of Fundamental Research, Mumbai 400005, India \\ ${ }^{100}$ INAF, Osservatorio Astronomico di Capodimonte, I-80131, Napoli, Italy \\ ${ }^{101}$ University of Michigan, Ann Arbor, Michigan 48109, USA \\ ${ }^{102}$ Rochester Institute of Technology, Rochester, New York 14623, USA \\ ${ }^{103}$ NCSA, University of Illinois at Urbana-Champaign, Urbana, Illinois 61801, USA \\ ${ }^{104}$ Universitat de les Illes Balears, IAC3-IEEC, E-07122 Palma de Mallorca, Spain \\ ${ }^{105}$ University of Biatystok, 15-424 Biatystok, Poland \\ ${ }^{106}$ SUPA, University of Strathclyde, Glasgow G1 1XQ, United Kingdom \\ ${ }^{107}$ IISER-TVM, CET Campus, Trivandrum Kerala 695016, India \\ ${ }^{108}$ Canadian Institute for Theoretical Astrophysics, University of Toronto, Toronto, \\ Ontario M5S 3H8, Canada \\ ${ }^{109}$ Institute of Applied Physics, Nizhny Novgorod, 603950, Russia \\ ${ }^{110}$ Pusan National University, Busan 609-735, Korea \\ ${ }^{111}$ Hanyang University, Seoul 133-791, Korea \\ ${ }^{112}$ University of Adelaide, Adelaide, South Australia 5005, Australia \\ ${ }^{113}$ NCBJ, 05-400 Świerk-Otwock, Poland \\ ${ }^{114}$ IM-PAN, 00-956 Warsaw, Poland \\ ${ }^{115}$ Monash University, Victoria 3800, Australia \\ ${ }^{116}$ Seoul National University, Seoul 151-742, Korea \\ ${ }^{117}$ The Chinese University of Hong Kong, Shatin, NT, Hong Kong \\ ${ }^{118}$ University of Alabama in Huntsville, Huntsville, Alabama 35899, USA \\ ${ }^{119}$ University of Massachusetts-Amherst, Amherst, Massachusetts 01003, USA \\ ${ }^{120}$ ESPCI, CNRS, F-75005 Paris, France \\ ${ }^{121}$ Università di Camerino, Dipartimento di Fisica, I-62032 Camerino, Italy \\ ${ }^{122}$ Southern University and A\&M College, Baton Rouge, Louisiana 70813, USA \\ ${ }^{123}$ College of William and Mary, Williamsburg, Virginia 23187, USA \\ ${ }^{124}$ Instituto de Física Teórica, University Estadual Paulista/ICTP South American Institute for \\ Fundamental Research, Sao Paulo SP 01140-070, Brazil \\ ${ }^{125}$ University of Cambridge, Cambridge CB2 1TN, United Kingdom \\ ${ }^{126}$ IISER-Kolkata, Mohanpur, West Bengal 741252, India \\ ${ }^{127}$ Rutherford Appleton Laboratory, HSIC, Chilton, Didcot, Oxon OX11 OQX, United Kingdom \\ ${ }^{128}$ Whitman College, 345 Boyer Avenue, Walla Walla, Washington 99362 USA \\ ${ }^{129}$ National Institute for Mathematical Sciences, Daejeon 305-390, Korea \\ ${ }^{130}$ Université de Lyon, F-69361 Lyon, France \\ ${ }^{131}$ Hobart and William Smith Colleges, Geneva, New York 14456, USA \\ ${ }^{132}$ Janusz, Gil Institute of Astronomy, University of Zielona Góra, 65-265 Zielona Góra, Poland \\ ${ }^{133}$ King's College London, University of London, London WC2R 2LS, United Kingdom \\ ${ }^{134}$ Andrews University, Berrien Springs, Michigan 49104, USA
}


${ }^{135}$ Università di Siena, I-53100 Siena, Italy

${ }^{136}$ Trinity University, San Antonio, Texas 78212, USA

${ }^{137}$ University of Washington, Seattle, Washington 98195, USA

${ }^{138}$ Kenyon College, Gambier, Ohio 43022, USA

${ }^{139}$ Abilene Christian University, Abilene, Texas 79699, USA

${ }^{140}$ Cornell Center for Astrophysics and Planetary Science, Cornell University, Ithaca, New York 14853, USA

${ }^{141}$ Theoretical Physics Institute, University of Jena, 07743 Jena, Germany

${ }^{142}$ Caltech JPL, Pasadena, California 91109, USA

${ }^{\dagger}$ Deceased. 